J. DIFFERENTIAL GEOMETRY

82 (2009) 467-500

\title{
CLIFFORD-WOLF HOMOGENEOUS RIEMANNIAN MANIFOLDS
}

\author{
Valerit N. Berestovskit \& Yurii G. Nikonorov
}

\begin{abstract}
In this paper, using connections between Clifford-Wolf isometries and Killing vector fields of constant length on a given Riemannian manifold, we classify simply connected Clifford-Wolf homogeneous Riemannian manifolds. We also get the classification of complete simply connected Riemannian manifolds with the Killing property defined and studied previously by J.E. D'Atri and H.K. Nickerson. In the last part of the paper we study properties of Clifford-Killing spaces, that is, real vector spaces of Killing vector fields of constant length, on odd-dimensional round spheres, and discuss numerous connections between these spaces and various classical objects.
\end{abstract}

\section{Introduction and the main result}

In this paper the authors give complete isometric classification of simply connected manifolds in the title. Recall that a Clifford-Wolf translation of a metric space is an isometry of the space onto itself moving all its points one and the same distance $[\mathbf{3 7}],[\mathbf{2 0}],[\mathbf{3 8}]$. A metric space is Clifford-Wolf homogeneous (or CW-homogeneous), if for any two its points there exists a Clifford-Wolf translation of the space moving one of these points to another [8].

It is not difficult to see that Euclidean spaces, odd-dimensional round spheres, and Lie groups with bi-invariant Riemannian metrics, as well as direct metric products of Clifford-Wolf homogeneous Riemannian spaces are Clifford-Wolf homogeneous. The main result of this paper states that in simply connected case the opposite statement is also true. More exactly,

The project is supported by the State Maintenance Program for the Leading Scientific Schools of the Russian Federation (grants NSH-5682.2008.1). The first author is supported in part by RFBR (grant 08-01-00067-a).

Received 04/05/2008. 
Theorem 1. A simply connected (connected) Riemannian manifold is Clifford-Wolf homogeneous if and only if it is a direct metric product of an Euclidean space, odd-dimensional spheres of constant curvature and simply connected compact simple Lie groups supplied with biinvariant Riemannian metrics (some of these factors could be absent).

In the first section we give in more details necessary definitions of spaces under investigation, discuss examples of Clifford-Wolf translations and groups consisting of them.

Clifford-Wolf homogeneous space is a special case of the so-called $\delta$ homogeneous metric space (see definition in Section 1 or in $[8]$ ). On the ground of the author's structure results on $\delta$-homogeneous Riemannian manifolds in [7], we present in the second section analogous results for CW-homogeneous manifolds.

In the third section, the authors elaborate main technical means and tools for the further investigation, namely Killing vector fields of constant length, their properties, and especially the very top result of Theorem 5 about the nullity of covariant derivative of the curvature tensor on Riemannian manifold when one uses three times one and the same Killing vector field of constant length.

The subject of the fourth section is well presented by its title. In particular, we find a connection of the notion of more general restrictively CW-homogeneous manifolds with the possibility of presenting geodesics as integral curves of Killing vector fields of constant length. With the help of authors' investigations of Killing vector fields on symmetric spaces in $[6]$, it is proved that for symmetric spaces this more general notion is equivalent to the old one.

In the next section the main result is proved. First of all, on the ground of mentioned Theorem 5 and one result in [10], we immediately get that any simply connected $\mathrm{CW}$-homogeneous Riemannian manifold is symmetric. After this, thoroughly, even not so long, study of the $\mathrm{CW}$-homogeneity condition in symmetric case permits to finish proof.

In Section 6 the Clifford-Killing spaces, that is, real vector spaces of Killing vector fields of constant length, are introduced.

Manifolds in the title of the seventh section have been defined and investigated by D'Atri and Nickerson in the paper [15]. They are exactly Riemannian manifolds which locally admit Clifford-Killing spaces of dimension equal to the dimension of the manifold. We proved in Theorem 11 that in simply connected complete case, these manifolds are classified similar to CW-homogeneous spaces in Theorem 1, but among odd-dimensional spheres one should leave only seven-dimensional one.

It is quite interesting that classical famous results of Radon and Hurwitz in [35] and [26], discussed in Section 8, can be interpreted exactly as a construction of Clifford-Killing spaces of maximal dimension on 
round odd-dimensional spheres, and famous Radon-Hurwitz function gives their dimensions.

In Section 9, a close connection of Clifford-Killing spaces on round odd-dimensional spheres with Clifford algebras and modules is established. This permits to classify all Clifford-Killing vector spaces on round spheres in Theorems 16 (and 17) up to self-isometries of spheres (preserving the orientation).

Radon in his paper [35] observed that his considerations of the Hurwitz Question 2 in Section 8 are closely connected with some (topological) spheres in the Lie group $O(2 n), n \geq 2$. In Section 10, we proved that any this Radon's sphere is a totaly geodesic sphere in $O(2 n)$ supplied with a bi-invariant Riemannian metric $\mu$.

In the next section we deal with more general question of totally geodesic spheres in $(S O(2 n), \mu)$ related to triple Lie systems in the Lie algebra of $S O(2 n)$ and Clifford-Killing spaces on round spheres $S^{2 n-1}$. In particular, it is proved, besides results, similar to results in previous section, that Radon's spheres coincide with totally geodesic Helgason's spheres (of constant sectional curvature $k$ equal to maximal sectional curvature of $(S O(2 n), \mu))$ from the paper [24] if and only if $n=2$. In Proposition 12, the curvature $k$ is calculated.

In the last section, Lie algebras in Clifford-Killing spaces on round odd-dimensional spheres are studied.

Acknowledgements. We thank P. Eberlein and J.A. Wolf for helpful discussion of this project.

\section{Preliminaries}

In the course of the paper, if the opposite is not stated, a Riemannian manifold means a connected $C^{\infty}$-smooth Riemannian manifold; the smoothness of any object means $C^{\infty}$-smoothness. For a Riemannian manifold $M$ and its point $x \in M$ by $M_{x}$ we denote the tangent (Euclidean) space to $M$ at the point $x$. For a given Riemannian manifold $(M, g)$ by $\rho$ we denote the inner (length) metric generated by the Riemannian metric tensor $g$ on $M$.

Recall that a Clifford-Wolf translation in $(M, g)$ is an isometry $s$ moving all points in $M$ one and the same distance, i.e. $\rho(x, s(x)) \equiv$ const for all $x \in M$. Notice that Clifford-Wolf translations are often called Clifford translations (see for example [37] or [44] or $[\mathbf{3 0}]$ ), but we follow in this case the terminology of the paper [20]. Clifford-Wolf translations naturally appear in the investigation of homogeneous Riemannian coverings of homogeneous Riemannian manifolds $[44,30]$. H. Freudenthal classified in the paper [20] all individual Clifford-Wolf translations on symmetric spaces. Let us indicate yet another construction of such transformations. Suppose that some isometry group $G$ acts transitively 
on a Riemannian manifold $M$ and $s$ is any element of the centralizer of $G$ in the full isometry group $\operatorname{Isom}(M)$ of $M$. Then $s$ is a Clifford-Wolf translation (in particular, if the center $Z$ of the group $G$ is not discrete, then every one-parameter subgroup in $Z$ consists of Clifford-Wolf translations on $M$ ). Indeed, if $x$ and $y$ are some points of manifold $M$, then there is $g \in G$ such that $g(x)=y$. Thus

$$
\rho(x, s(x))=\rho(g(x), g(s(x)))=\rho(g(x), s(g(x)))=\rho(y, s(y)) .
$$

For symmetric spaces, this result can be inverted due to V. Ozols (see [34], Corollary 2.7): If $M$ is symmetric, then for any Clifford-Wolf translation $s$ on $M$, the centralizer of $s$ in $\operatorname{Isom}(M)$ acts transitively on $M$.

Notice that several classical Riemannian manifolds possesses a oneparameter group of Clifford-Wolf translations. For instance, one knows that among irreducible compact simply connected symmetric spaces only odd-dimensional spheres, the spaces $S U(2 m) / S p(m), m \geq 2$, and simple compact Lie groups, supplied with some bi-invariant Riemannian metric, admit one-parameter groups of Clifford-Wolf translations [39]. Recall, that an inner metric space $(M, \rho)$ is a metric space $M$ with distance $\rho$ such that for all $x, y \in M, \rho(x, y)$ is the infimum of the lengths of curves $\alpha$ joining $x$ and $y$ in $M$.

Definition $1([\mathbf{8}])$. An inner metric space $(M, \rho)$ is called CliffordWolf homogeneous if for every two points $y, z$ in $M$ there exists a Clifford-Wolf translation of the space $(M, \rho)$ moving $y$ to $z$.

Let us consider some examples. Obviously, every Euclidean space $\mathbb{E}^{n}$ is Clifford-Wolf homogeneous. Since $\mathbb{E}^{n}$ can be treated as a (commutative) additive vector group with a bi-invariant inner product, the following example can be considered as a generalization.

Example 1. Let $G$ be a Lie group supplied with a bi-invariant Riemannian metric $\rho$. In this case both the group of left shifts $L(G)$ and the group of right shifts $R(G)$ consist of Clifford-Wolf isometries of $(G, \rho)$. Therefore, $(G, \rho)$ is Clifford-Wolf homogeneous. Note also that in [5] the following result has been proved: A Riemannian manifold $(M, g)$ admits a transitive group $\Gamma$ of Clifford-Wolf translations if and only if it is isometric to some Lie group $G$ supplied with a bi-invariant Riemannian metric.

Example 2. Every odd-dimensional round sphere $S^{2 n-1}$ is CliffordWolf homogeneous. Indeed,

$$
S^{2 n-1}=\left\{\xi=\left(z_{1}, \ldots, z_{n}\right) \in \mathbb{C}^{n}: \sum_{k=1}^{n}\left|z_{k}\right|^{2}=1\right\} .
$$

Then the formula $\gamma(t)(\xi)=e^{i t} \xi$ defines a one-parameter group of Clifford-Wolf translations on $S^{2 n-1}$ with all orbits as geodesic circles. Now, 
since $S^{2 n-1}$ is homogeneous and isotropic, any its geodesic circle is an orbit of a one-parameter group of Clifford-Wolf translations, and so $S^{2 n-1}$ is Clifford-Wolf homogeneous. Note that $S^{1}$ and $S^{3}$ can be treated as the Lie groups $S O(2)$ and $S U(2)$ with bi-invariant Riemannian metrics.

Note also that any direct metric product of Clifford-Wolf homogeneous Riemannian manifolds is Clifford-Wolf homogeneous itself. On the other hand, the condition for a Riemannian manifold to be CliffordWolf homogeneous, is quite strong. Therefore, one should hope to get a complete classification of such manifolds.

Definition 2. An inner metric space $(M, \rho)$ is called restrictively Clifford-Wolf homogeneous if for any $x \in M$ there exists a number $r(x)>0$ such that for any two points $y, z$ in open ball $U(x, r(x))$ there exists a Clifford-Wolf translation of the space $(M, \rho)$ moving $y$ to $z$.

The notion of (restrictively) Clifford-Wolf homogeneous metric space is related to the notion of $\delta$-homogeneous metric space. Recall the following definition.

Definition $3([8])$. Let $(X, d)$ be a metric space and $x \in X$. An isometry $f: X \rightarrow X$ is called $a \delta(x)$-translation, if $x$ is a point of maximal displacement of $f$, i.e. for every $y \in X$ the relation $d(y, f(y)) \leq$ $d(x, f(x))$ holds. A metric space $(X, d)$ is called $\delta$-homogeneous, if for every $x, y \in X$ there exists a $\delta(x)$-translation of $(X, d)$, moving $x$ to $y$.

It is easy to see that every restrictively Clifford-Wolf homogeneous locally compact complete inner metric space is $\delta$-homogeneous (see Proposition 1 in [7]). Therefore, we can apply all results on $\delta$-homogeneous Riemannian manifolds to (restrictively) Clifford-Wolf homogeneous Riemannian manifolds [7].

We shall need also the following

Definition 4. An inner metric space $(X, d)$ is called strongly CliffordWolf homogeneous if for every two points $x, y \in X$ there is a oneparameter group $\gamma(t), t \in \mathbb{R}$, of Clifford-Wolf translations of the space $(X, d)$ such that for sufficiently small $|t|, \gamma(t)$ shifts all points of $(X, d)$ to distance $|t|$, and $\gamma(s)(x)=y$, where $d(x, y)=s$.

It is clear that any strongly Clifford-Wolf homogeneous inner metric space is a Clifford-Wolf homogeneous and Clifford-Wolf homogeneous inner metric space is a restrictively Clifford-Wolf homogeneous. It is a natural question: are the above three classes pairwise distinct (in particular, in the case of Riemannian manifolds)? 


\section{Some structure results}

Lemma 1. A Clifford-Wolf isometry of a metric space $(X, d)$ is a $\delta(x)$-translation for every $x \in X$. In particular a Clifford-Wolf homogeneous Riemannian manifold is $\delta$-homogeneous. Further, every connected restrictively Clifford-Wolf homogeneous Riemannian manifold is homogeneous and thus is complete.

Proof. For the first statement, a Clifford-Wolf isometry $f$ has constant displacement so $d(x, f(x))=d(y, f(y))$ for all $x, y \in X$. For the last statement, the orbits of the isometry group all are open so by connectedness there is only one orbit.

q.e.d.

Theorem 2. Every (restrictively) Clifford-Wolf homogeneous Riemannian manifolds is $\delta$-homogeneous, has non-negative sectional curvature, and is a direct metric product of Euclidean space and a compact (restrictively) Clifford-Wolf homogeneous Riemannian manifold.

Proof. The first assertion is a part of Lemma 1. The second one follows from the fact that every $\delta$-homogeneous Riemannian manifold has nonnegative sectional curvature (see [7] and [8] for some more general results). The third assertion easily follows from Toponogov's theorem in $[\mathbf{3 6}]$, stating that every complete Riemannian manifold $(M, \mu)$ with nonnegative sectional curvature, containing a metric line, is isometric to a direct Riemannian product $(N, \nu) \times \mathbb{R}$ (more general results in this direction could be found in [8]) and the fact that any Clifford-Wolf translation preserves a product structure on a metric product of two Riemannian manifolds, and so is a direct product of suitable CliffordWolf translations on these manifolds (see Theorem 3.1.2 in [39]). q.e.d.

Theorem 3 (Corollary 3 in [7]). Any Riemannian covering of a (restrictively) Clifford-Wolf homogeneous Riemannian manifold, is (restrictively) Clifford-Wolf homogeneous itself.

Theorem 4. Let $M$ be a simply connected (restrictively) CliffordWolf homogeneous Riemannian manifold and $M=M_{0} \times M_{1} \times \cdots \times M_{k}$ its de Rham decomposition, where $M_{0}$ is Euclidean space, and the others $M_{i}, 1 \leq i \leq k$, are simply connected compact Riemannian manifolds. Then every $M_{i}$ is a (restrictively) Clifford-Wolf homogeneous Riemannian manifolds. Moreover, any isometry $f$ of $M$ is a Clifford-Wolf translation if and only if it is a product of some Clifford-Wolf translations $f_{i}$ on $M_{i}$.

Proof. Obviously, the first assertion is a consequence of the second one, that was proved in Corollary 3.1.3 in [39]. $\quad$ q.e.d. 


\section{On Killing vector fields of constant length}

Here we consider some properties of Killing vector fields of constant length on Riemannian manifolds. Recall that a vector field $X$ on a Riemannian manifold $(M, g)$ is called Killing if $L_{X} g=0$. For a Killing vector field $X$ it is useful to consider the operator $A_{X}$ defined on vector fields by the formula $A_{X} V=-\nabla_{V} X$. It is clear that $A_{X}=L_{X}-\nabla_{X}$.

All assertions of the following lemma are well known (see e.g. [30]).

Lemma 2. Let $X$ be a Killing vector field on the Riemannian manifold $(M, g)$. Then the following statements hold:

1) For any vector fields $U$ and $V$ on $M$ the equality $g\left(\nabla_{U} X, V\right)+$ $g\left(U, \nabla_{V} X\right)=0$ holds. In other words, the operator $A_{X}$ is skew-symmetric.

2) For every vector field $U$ on $M$ the equality

$$
R(X, U)=\left[\nabla_{X}, \nabla_{U}\right]-\nabla_{[X, U]}=\left[\nabla_{U}, A_{X}\right]
$$

holds, where $R$ is the curvature tensor of $(M, g)$.

3) For any Killing vector field $X$ and for any vector fields $U, V, W$ on a Riemannian manifold $(M, g)$ the following formula holds:

$$
-g(R(X, U) V, W)=g\left(\nabla_{U} \nabla_{V} X, W\right)+g\left(\nabla_{U} V, \nabla_{W} X\right),
$$

where $R$ is the curvature tensor of $(M, g)$.

Proof. It is clear that $X \cdot g(U, V)=g\left(\nabla_{X} U, V\right)+g\left(U, \nabla_{X} V\right)$ and $X \cdot g(U, V)=g([X, U], V)+g(U,[X, V])$. Therefore,

$g\left(\nabla_{U} X, V\right)+g\left(U, \nabla_{V} X\right)=g\left(\nabla_{X} U-[X, U], V\right)+g\left(U, \nabla_{X} V-[X, V]\right)=0$, that proves the first assertion.

The second assertion is proved in Lemma 2.2 of [31] (see also Proposition 2.2 of Chapter 6 in $[\mathbf{3 0}])$. The third assertion follows from the previous one:

$$
\begin{gathered}
g(R(X, U) V, W)=g\left(\nabla_{U} A_{X} V, W\right)-g\left(A_{X} \nabla_{U} V, W\right)= \\
-g\left(\nabla_{U} \nabla_{V} X, W\right)+g\left(\nabla_{U} V, A_{X} W\right)=-g\left(\nabla_{U} \nabla_{V} X, W\right)-g\left(\nabla_{U} V, \nabla_{W} X\right),
\end{gathered}
$$

because the operator $A_{X}$ is skew symmetric for a Killing vector field $X$.

q.e.d.

Now we recall some well known properties of Killing vector field of constant length.

Lemma 3. Let $X$ be a Killing vector field $X$ on a Riemannian manifold $(M, g)$. Then the following conditions are equivalent:

1) $X$ has constant length on $M$;

2) $\nabla_{X} X=0$ on $M$;

3) every integral curve of the field $X$ is a geodesic in $(M, g)$. 
Proof. It suffices to note that the length of $X$ is constant along any its integral curve, and for any Killing vector field $X$ and arbitrary smooth vector field $Y$ on $(M, g)$ we have the following equality:

$$
\begin{gathered}
0=\left(L_{X} g\right)(X, Y)=X \cdot g(X, Y)-g([X, X], Y)-g(X,[X, Y])= \\
g\left(\nabla_{X} X, Y\right)+g\left(X, \nabla_{X} Y\right)-g(X,[X, Y])= \\
g\left(\nabla_{X} X, Y\right)+g\left(X, \nabla_{Y} X\right)=g\left(\nabla_{X} X, Y\right)+\frac{1}{2} Y \cdot g(X, X) .
\end{gathered}
$$

q.e.d.

Proposition 1. Let $Z$ be a Killing vector field of constant length and $X, Y$ arbitrary vector fields on a Riemannian manifold $(M, g)$. Then the formula

$$
g\left(\nabla_{X} Z, \nabla_{Y} Z\right)=g(R(X, Z) Z, Y)=g(R(Z, Y) X, Z)
$$

holds on $M$.

Proof. Since $g(Z, Z)=$ const, then $X \cdot g(Z, Z)=2 g\left(\nabla_{X} Z, Z\right)=0$. Therefore,

$$
0=Y \cdot g\left(\nabla_{X} Z, Z\right)=g\left(\nabla_{Y} \nabla_{X} Z, Z\right)+g\left(\nabla_{X} Z, \nabla_{Y} Z\right) .
$$

By Assertion 3) in Lemma 2 we get

$$
\begin{aligned}
g\left(\nabla_{Y} \nabla_{X} Z, Z\right) & =-g(R(Z, Y) X, Z)-g\left(\nabla_{Y} X, \nabla_{Z} Z\right) \\
& =-g(R(Z, Y) X, Z),
\end{aligned}
$$

because $\nabla_{Z} Z=0$. Therefore, $g\left(\nabla_{X} Z, \nabla_{Y} Z\right)=g(R(Z, Y) X, Z)$. The formula $g\left(\nabla_{X} Z, \nabla_{Y} Z\right)=g(R(X, Z) Z, Y)$ follows from symmetries of the curvature tensor.

q.e.d.

Lemma 4. For every Killing vector field of constant length $Z$ and any vector fields $X, Y$ on a Riemannian manifold $(M, g)$ the following equalities hold:

$$
\begin{gathered}
g\left(R(X, Z) Z, \nabla_{Y} Z\right)+g\left(R(Y, Z) Z, \nabla_{X} Z\right)=0, \\
g\left(\nabla_{Z} \nabla_{Y} Z, \nabla_{X} Z\right)=g\left(R(X, Z) Z, \nabla_{Z} Y\right) .
\end{gathered}
$$

Proof. Let us prove the first equality. By Proposition 1 we have

$$
\begin{gathered}
g\left(R(X, Z) Z, \nabla_{Y} Z\right)+g\left(R(Y, Z) Z, \nabla_{X} Z\right)= \\
g\left(\nabla_{X} Z, \nabla_{\nabla_{Y} Z} Z\right)+g\left(\nabla_{Y} Z, \nabla_{\nabla_{X} Z} Z\right)=g\left(U, \nabla_{V} Z\right)+g\left(V, \nabla_{U} Z\right),
\end{gathered}
$$

where $U=\nabla_{X} Z$ and $V=\nabla_{Y} Z$. Now, using Assertion 1) in Lemma 2, we get $g\left(U, \nabla_{V} Z\right)+g\left(V, \nabla_{U} Z\right)=0$.

Further, since $\nabla_{Z} Z=0$ and for any vector field $W$ the equalities

$$
\nabla_{Z} \nabla_{W}=\nabla_{W} \nabla_{Z}+\nabla_{[Z, W]}+R(Z, W), \quad \nabla_{Z} W=\nabla_{W} Z+[Z, W]
$$

hold, we get (using Proposition 1)

$$
g\left(\nabla_{Z} \nabla_{Y} Z, \nabla_{X} Z\right)=g\left(\nabla_{[Z, Y]} Z, \nabla_{X} Z\right)+g\left(R(Z, Y) Z, \nabla_{X} Z\right)=
$$




$$
\begin{gathered}
g(R(X, Z) Z,[Z, Y])+g\left(R(Z, Y) Z, \nabla_{X} Z\right)= \\
g\left(R(X, Z) Z, \nabla_{Z} Y\right)-g\left(R(X, Z) Z, \nabla_{Y} Z\right)-g\left(R(Y, Z) Z, \nabla_{X} Z\right) .
\end{gathered}
$$

On the other hand, we have proved that

$$
g\left(R(X, Z) Z, \nabla_{Y} Z\right)+g\left(R(Y, Z) Z, \nabla_{X} Z\right)=0,
$$

hence we get the second equality.

q.e.d.

Now we can prove the following theorem, that plays a key role in our study.

Theorem 5. For any Killing vector field of constant length $Z$ on a Riemannian manifold $(M, g)$,

$$
\left(\nabla_{Z} R\right)(\cdot, Z) Z \equiv 0 .
$$

Proof. It suffices to prove that $g\left(\left(\nabla_{Z} R\right)(X, Z) Z, Y\right)=0$ for every vector fields $X$ and $Y$ on $M$. From Proposition 1 we know that

$$
g\left(\nabla_{X} Z, \nabla_{Y} Z\right)=g(R(X, Z) Z, Y) .
$$

Therefore,

$$
Z \cdot g\left(\nabla_{X} Z, \nabla_{Y} Z\right)=Z \cdot g(R(X, Z) Z, Y) .
$$

Further, by Lemma 4, we get that

$$
\begin{gathered}
Z \cdot g\left(\nabla_{X} Z, \nabla_{Y} Z\right)=g\left(\nabla_{Z} \nabla_{X} Z, \nabla_{Y} Z\right)+g\left(\nabla_{X} Z, \nabla_{Z} \nabla_{Y} Z\right)= \\
g\left(R(Y, Z) Z, \nabla_{Z} X\right)+g\left(R(X, Z) Z, \nabla_{Z} Y\right) .
\end{gathered}
$$

On the other hand,

$$
\begin{gathered}
Z \cdot g(R(X, Z) Z, Y)=g\left(\left(\nabla_{Z} R\right)(X, Z) Z, Y\right)+ \\
g\left(R\left(\nabla_{Z} X, Z\right) Z, Y\right)+g\left(R(X, Z) Z, \nabla_{Z} Y\right)= \\
g\left(\left(\nabla_{Z} R\right)(X, Z) Z, Y\right)+g\left(R(Y, Z) Z, \nabla_{Z} X\right)+g\left(R(X, Z) Z, \nabla_{Z} Y\right) .
\end{gathered}
$$

Combining the equations above, we get $g\left(\left(\nabla_{Z} R\right)(X, Z) Z, Y\right)=0$, that proves the theorem.

q.e.d.

Note that the condition $\left(\nabla_{Z} R\right)(\cdot, Z) Z=0$ means that for any geodesic $\gamma$ that is an integral curve of the field $Z$, a derivative of any normal Jacobi field along $\gamma$ is also a normal Jacobi field (see Section 2.33 in $[\mathbf{1 0}])$. 


\section{Interrelations of Clifford-Wolf isometries and Killing fields with constant length}

There exists a connection between Killing vector fields of constant length and Clifford-Wolf translations in a Riemannian manifold $(M, g)$. The following proposition is evident.

Proposition 2. Suppose that a one-parameter isometry group $\gamma(t)$ on $(M, g)$, generated by a Killing vector field $X$, consists of CliffordWolf translations. Then $X$ has constant length.

Proposition 2 can be partially inverted. More exactly, we have

Proposition 3 ([6]). Suppose a Riemannian manifold $(M, g)$ has the injectivity radius, bounded from below by some positive constant (in particularly, this condition is satisfied for arbitrary compact or homogeneous manifold), and $X$ is a Killing vector field on $(M, g)$ of constant length. Then isometries $\gamma(t)$ from the one-parameter motion group, generated by the vector field $X$, are Clifford-Wolf translations if $t$ is close enough to 0 .

Theorem $6([\mathbf{7}])$. Let $(M, g)$ be a compact homogeneous Riemannian manifold. Then there exists a positive number $s>0$ such that for arbitrary motion $f$ of the space $(M, g)$ with maximal displacement $\delta$, which is less than $s$, there is a unique Killing vector field $X$ on $(M, g)$ such that $\max _{x \in M} \sqrt{g(X(x), X(x))}=1$ and $\gamma_{X}(\delta)=f$, where $\gamma_{X}(t)$, $t \in \mathbb{R}$, is the one-parameter motion group of $(M, \mu)$, generated by the field $X$. If moreover $f$ is a Clifford-Wolf translation, then the Killing vector field $X$ has unit length on $(M, g)$.

From previous results we easily get the following

Theorem 7. A Riemannian manifold $(M, g)$ is restrictively CliffordWolf homogeneous if and only if it is complete and every geodesic $\gamma$ in $(M, g)$ is an integral curve of a Killing vector field of constant length on $(M, g)$.

Proof. Since by Theorem 2 every restrictively Clifford-Wolf homogeneous Riemannian manifold is a direct metric product of Euclidean space and a compact restrictively Clifford-Wolf homogeneous Riemannian manifold, it is sufficient to consider the compact case.

Suppose that $(M, g)$ is restrictively Clifford-Wolf homogeneous. Let us take any $x \in M$ and any geodesic $\gamma$ through the point $x$. Consider the number $r(x)>0$ as in Definition 2, and take any point $y \in \gamma$, $y \neq x$, such that $\varepsilon:=\rho(x, y)<\min \left\{r(x), r_{\text {inj }}(x), s\right\}$, where $r_{\text {inj }}(x)$ is the injectivity radius at $x$ and $s$ is taken from the statement of Theorem 6 . Then by Definition 2 there is a Clifford-Wolf translation moving $x$ to $y$. By Theorem 6 , there is a unit Killing field $X$ on $(M, g)$ such that $\gamma_{X}(\varepsilon)=f$, where $\gamma_{X}(t), t \in \mathbb{R}$ is the one-parameter motion group 
generated by the field $X$. By Lemma 3 an integral curve of $X$ through $x$ is a geodesic, that evidently coincides with $\gamma$. Since $x$ is an arbitrary point of $M$, then any geodesic $\gamma$ on $(M, g)$ is an integral curve of a Killing field of constant length on $(M, g)$.

Now, suppose that every geodesic $\gamma$ on $(M, g)$ is an integral curve of a Killing field of constant length on $(M, g)$. By Proposition 3 there is $\delta>0$ such that for any unit Killing vector field $X$ on $(M, g)$, all isometries $\gamma(t),|t|<\delta$, from the one-parameter motion group, generated by $X$, are Clifford-Wolf translations. Now, for any $x, y \in M$ such that $\rho(x, y)<\delta$, there is a geodesic $\gamma$ through $x$ and $y$ such that the segment between $x$ and $y$ has length $\rho(x, y)$. Let $X$ be a unit Killing field such that $\gamma$ is an integral curve of $X$. Consider a one-parameter motion group $\gamma(t)$, $t \in \mathbb{R}$, generated by $X$. Then $s_{1}=\gamma(\rho(x, y))$ and $s_{2}=\gamma(-\rho(x, y))$ are Clifford-Wolf isometries on $(M, g)$, and either $s_{1}(x)=y$ or $s_{2}(x)=y$. Therefore, $(M, g)$ is restrictively Clifford-Wolf homogeneous. q.e.d.

Let us cite the following result.

Theorem 8 ([6]). Let $M$ be a symmetric Riemannian space, $X$ is a Killing vector field of constant length on $M$. Then the one-parameter isometry group $\mu(t), t \in \mathbb{R}$, of the space $M$, generated by the field $X$, consists of Clifford-Wolf translations. Moreover, if the space $M$ has positive sectional curvature, then the flow $\mu(t), t \in \mathbb{R}$, admits a factorization up to a free isometric action of the circle $S^{1}$ on $M$.

From Theorems 7 and 8, we get the following

Theorem 9. If a restrictively Clifford-Wolf homogeneous Riemannian manifold $(M, \mu)$ is symmetric space, then it is strongly Clifford-Wolf homogeneous.

Proof. Let $x, y$ be arbitrary points in $(M, \mu)$. Take a shortest geodesic $\gamma$ in $(M, g)$, joining points $x$ and $y$. Then by Theorem 7 , the geodesic $\gamma$ parameterized by the arclength is an integral curve of a unit Killing vector field $X$ on $(M, g)$. Now, by Theorem 8 , the one-parameter isometry group $\mu(t), t \in \mathbb{R}$, of the space $M$, generated by the field $X$, consists of Clifford-Wolf translations. It is clear that for sufficiently small $|t|$, $\gamma(t)$ shifts all points of $(X, d)$ to distance $|t|$, and $\gamma(s)(x)=y$, where $d(x, y)=s$.

q.e.d.

\section{Proof of the main result}

We shall need the following useful proposition.

Proposition 4 (Proposition 2.35 in [10]). If the Levi-Civita derivative of the curvature tensor $R$ of a Riemannian manifold $(M, g)$ satisfies the condition $\left(\nabla_{X} R\right)(\cdot, X) X=0$ for any $X \in T M$, then $(M, g)$ is locally symmetric. 
Now, using Theorem 5 we can prove the following

Theorem 10. Every restrictively Clifford-Wolf homogeneous Riemannian manifold $(M, g)$ is locally symmetric.

Proof. According to Proposition 4, in order to prove that $(M, g)$ is locally symmetric it suffices to prove $\left(\nabla_{X} R\right)(\cdot, X) X=0$ for any $X \in M_{x}$ at a fixed point $x \in M$. By Theorem 7 any geodesic $\gamma$ on $(M, g)$ is an integral curve of a Killing vector field of constant length on $(M, g)$, hence, we can find a Killing field of constant length $Z$ on $(M, g)$ such that $Z(x)=X$. By Theorem 5 we get $\left(\nabla_{Z} R\right)(\cdot, Z) Z=0$ at every point of $M$. In particular,

$$
\left(\nabla_{X} R\right)(\cdot, X) X=\left(\nabla_{Z(x)} R\right)(\cdot, Z(x)) Z(x)=0 .
$$

The theorem is proved.

q.e.d.

Proposition 5 (Theorem 5.5.1 in [39]). Let $M$ be a simply connected compact irreducible symmetric space, that is not isometric to a Lie group with a bi-invariant Riemannian metric. If $M$ admits nontrivial Killing vector field of constant length $X$, then either $M$ is an odd-dimensional sphere $S^{2 n-1}$ for $n \geq 3$, or $M=S U(2 n) / S p(n), n \geq 3$.

Proof. The assertion of the Proposition follows from Theorem 5.5.1 in [39], where it is proved that among the irreducible compact simply connected symmetric spaces only odd-dimensional spheres, spaces $S U(2 m) / S p(m), m \geq 2$, and compact simple Lie groups with bi-invariant Riemannian metrics, admit one-parameter groups of Clifford-Wolf translations. Now we give a somewhat different proof of Proposition 5 .

Let $G$ be the identity component of the full isometry group of $M$. Consider a one-parameter isometry group $\mu(t), t \in \mathbb{R}$, generated by $X$ (in fact, this group consists of Clifford-Wolf translations of $M[6]$ ). By Lemma 1 in [6] $Z_{\mu}$, the centralizer of the flow $\mu$ in $G$, acts transitively on $M$ (note that this result is based on some results of V. Ozols [34]). It is clear that the identity component $K=K\left(Z_{\mu}\right)$ of $Z_{\mu}$ is a Lie group, which acts transitively on $M$ and has a non-discrete center (this center contains $\mu(t), t \in \mathbb{R})$.

Note that $M=G / H$ is a homogeneous space, where $H$ is the isotropy subgroup at some point $x \in M$. Moreover, according to assumptions of the theorem, $G$ and $H$ are connected (recall that $M$ is simply connected) and $G$ is a simple compact Lie group.

In Theorem 4.1 of the paper [33], A.L. Onishchik classified all connected proper subgroups $K$ of the group $G$, that act transitively on the homogeneous space $G / H$, where $G$ is a simple compact connected Lie group and $H$ is its connected closed subgroup. If in this situation $K$ has a non-discrete center, then this theorem implies that either $G / H$ is an odd-dimensional sphere, or $G / H=S U(2 n) / S p(n), n \geq 3$. Since the center of $K\left(Z_{\mu}\right)$ is non-discrete, it proves the proposition. q.e.d. 
Proposition 6. Let $M$ be a symmetric space $S U(2 n) / S p(n)$, where $n \geq 3$. Then every Killing vector field of constant length on $M$ has the form $\operatorname{Ad}(s)(t U)$, where $U=\sqrt{-1} \operatorname{diag}(1,1, \ldots, 1,-(2 n-1)) \in \operatorname{su}(2 n)$, $s \in S U(2 n), t \in \mathbb{R}$. Moreover, $M$ is not restrictively Clifford-Wolf homogeneous.

Proof. We reformulate the calculation on page 89 of [39]. According to Theorem 4.1 in [33], there is a unique (up to conjugation in $S U(2 n)$ ) connected subgroup with non-discrete center $K \subset S U(2 n)$ that acts transitively on the homogeneous space $M=S U(2 n) / S p(n)$ $(n \geq 3)$. This is the group $S U(2 n-1) \times S^{1}$, where $S U(2 n-1)$ is embedded in $S U(2 n)$ via $A \rightarrow \operatorname{diag}(A, 1)$ and $S^{1}=\exp (t U), t \in \mathbb{R}$, $U=\sqrt{-1} \operatorname{diag}(1,1, \ldots, 1,-(2 n-1)) \in s u(2 n)$.

If $S U(2 n-1) \times S^{1}$ is the centralizer of a Killing field $V \in s u(2 n)$, then clearly $V=t U$ for a suitable $t \in \mathbb{R}$. It is clear also that any such Killing field has constant length on $M$, since it lies in the center of the Lie algebra of the group acting transitively on $M$. This proves the first assertion of the proposition.

Note that $\operatorname{dim}(S U(2 n) / S p(n))=(n-1)(2 n+1)$. On the other hand, we can easily calculate the dimension of the set of Killing fields of constant length on $M$. Indeed, this set is $\operatorname{Ad}(S U(2 n))(t U)$ (the orbit of $t U \in s u(2 n)$ under the adjoin action of the group $S U(2 n))$, $t \in \mathbb{R}$. For any fixed $t$ this orbit is $S U(2 n) / S(U(2 n-1) \cdot U(1))$, and $\operatorname{dim}(S U(2 n) / S(U(2 n-1) \cdot U(1)))=4 n-2$. Since $(4 n-2)+1<$ $(n-1)(2 n+1)=\operatorname{dim}(M)$ for $n \geq 3$, then $M$ is not restrictively Clifford-Wolf homogeneous. Otherwise, by Theorem 7 for any $x \in M$ and $U \in M_{x}$ there is a Killing vector field of constant length $X$ on $M$ such that $X(x)=U$, which is impossible by previous calculation of dimensions.

q.e.d.

Now we can prove the main result of the paper.

Proof of Theorem 1. Let $M$ be a simply connected Clifford-Wolf homogeneous Riemannian manifold. By Lemma $1 M$ is complete. Therefore, by Theorem $10 M$ is symmetric space. Let us consider the de Rham decomposition

$$
M=M_{0} \times M_{1} \times \cdots \times M_{k},
$$

where $M_{0}$ is Euclidean space, and the others $M_{i}, 1 \leq i \leq k$, are simply connected compact irreducible symmetric spaces. By Theorem 4, every $M_{i}, 0 \leq i \leq k$, is Clifford-Wolf homogeneous.

From Propositions 5 and 6 we get that every simply connected compact irreducible symmetric space that is Clifford-Wolf homogeneous is either an odd-dimensional sphere of constant curvature, or a simply connected compact simple Lie group supplied with a bi-invariant Riemannian metric. This proves the necessity. 
On the other hand, a direct metric product of an Euclidean space, odd-dimensional spheres of constant curvature and simply connected compact simple Lie groups supplied with bi-invariant Riemannian metrics (all these manifolds are Clifford-Wolf homogeneous) is a CliffordWolf homogeneous Riemannian manifold.

q.e.d.

\section{Clifford-Killing spaces}

The following proposition is evident.

Proposition 7. A collection $\left\{X_{1}, \ldots, X_{l}\right\}$ of Killing vector fields on a Riemannian manifold $(M, g)$ constitutes a basis of a finite-dimensional vector space $C K_{l}$ (over $\mathbb{R}$ ) of Killing vector fields of constant length if and only if vector fields $X_{1}, \ldots, X_{l}$ are linearly independent and all inner products $g\left(X_{i}, X_{j}\right) ; i, j=1, \ldots, l$ are constant. Under this $C K_{l}$ admits an orthonormal basis of (unit) Killing vector fields.

We shall call such a space $C K_{l}, l \geq 1$, a Clifford-Killing space or simply $C K$-space. Below we give a simple method to check the condition $g(X, Y)=$ const for given Killing vector fields $X, Y$.

Lemma 5. Suppose $X$ and $Y$ are Killing fields on a Riemannian manifold $(M, g)$. Then a point $x \in M$ is a critical point of the function $x \mapsto g_{x}(X, Y)$ if and only if

$$
\nabla_{X} Y=-\nabla_{Y} X=\frac{1}{2}[X, Y]
$$

at this point.

Proof. Since $\nabla_{X} Y-\nabla_{Y} X=[X, Y]$, it suffices to prove that $x$ is a critical point of $g(X, Y)$ if and only if $\nabla_{X} Y+\nabla_{Y} X=0$ at the point $x$. For any Killing vector field $W$ and arbitrary vector fields $U$ and $V$ on $(M, g)$ we have the equality $g\left(\nabla_{U} W, V\right)+g\left(U, \nabla_{V} W\right)=0$. Since $X$ and $Y$ are Killing vector fields, for any vector field $Z$ we get at the point $x$

$$
\begin{gathered}
0=Z \cdot g(X, Y)=g\left(\nabla_{Z} X, Y\right)+g\left(X, \nabla_{Z} Y\right)= \\
-g\left(Z, \nabla_{Y} X\right)-g\left(\nabla_{X} Y, Z\right)=-g\left(Z, \nabla_{Y} X+\nabla_{X} Y\right),
\end{gathered}
$$

that proves the lemma.

q.e.d.

Corollary 1. Suppose $X$ and $Y$ are Killing vector fields on a Riemannian manifold $(M, g)$. Then $g(X, Y)=$ const if and only if

$$
\nabla_{X} Y=-\nabla_{Y} X=\frac{1}{2}[X, Y] .
$$

In particular, a Killing vector field $X$ has constant length if and only if $\nabla_{X} X=0$.

Definition 5. Let $V$ and $W$ be some CK-spaces on $(M, g)$. We say that $V$ is (properly) equivalent to $W$ if there exists a (preserving orientation) isometry $f$ of $(M, g)$ onto itself such that $d f(V)=W$. 
The following question is quite interesting.

Question 1. Classify all homogeneous Riemannian manifolds which admit nontrivial CK-spaces. For any such manifold, classify up to (proper) equivalence all (in particular all maximal by inclusion) possible CK-spaces.

This difficult question mainly has not been considered before. All consequent sections are related to this question. We shall see that it is closely connected with some impressive classical and recent results.

\section{Riemannian manifolds with the Killing property}

In the paper [15], J.E. D'Atri and H.K. Nickerson studied Riemannian manifolds with the Killing property.

Definition 6 ([15]). A Riemannian manifold $(M, g)$ is said to have the Killing property if, in some neighborhood of each point of $M$, there exists an orthonormal frame $\left\{X_{1}, \ldots, X_{n}\right\}$ such that each $X_{i}$, $i=1, \ldots, n$, is a Killing vector field (local infinitesimal isometry). Such a frame is called a Killing frame.

Remark 1. We shall call also the property from Definition 6 by "the local Killing property", while a Riemannian manifold which has a globally defined Killing frame will be considered as "a manifold with the global Killing property". Note that a global Killing frame on $(M, g)$ defines an absolute parallelism in the sense of Cartan and Schouten $[\mathbf{1 2}]$. In fact, even in the indefinite metric setting it defines an absolute parallelism consistent with the Riemannian structure; see [42] and [43].

It is easy to see that every Lie group supplied with a bi-invariant Riemannian metric has the (global) Killing property. Note that a generalization of the Killing property is the divergence property, that we shall not treat in this paper (see details in [15]).

Proposition 8 ([15]). Every Riemannian manifold with the local Killing property is locally symmetric.

Proof. We prove the proposition by the method different from the another one in [15]. By definition, for any point $x$ in a given manifold $(M, g)$ there is a Killing frame $\left\{X_{1}, \ldots, X_{n}\right\}$ in some neighborhood $U$ of the point $x$. Since $g\left(X_{i}, X_{j}\right)=\delta_{i j}$, then for any real constants $a_{i}$, the local vector field $a_{1} X_{1}+a_{2} X_{2}+\cdots+a_{n} X_{n}$ is a local Killing field of constant length. As a corollary, for any vector $v \in M_{x}$ there is a Killing field $Z$ of constant length in $U$ such that $Z(x)=v$. Then, by the proof of Theorem 5 , which really doesn't require a global character of vector fields, we get that $\left(\nabla_{Z} R\right)(\cdot, Z) Z(x)=0$ or $\left(\nabla_{v} R\right)(\cdot, v) v=0$. By Proposition $4,(M, g)$ is locally symmetric.

q.e.d. 
Remark 2. Another proof of the previous proposition is given in [42]. The manifold $(M, g)$ has a (locally defined) consistent absolute parallelism defined by a local Killing frame, so it is locally symmetric by Theorem 4.14 in $[\mathbf{4 2}]$.

It is well known that every (not necessarily complete) locally symmetric Riemannian manifold is locally isometric to a symmetric space (see e.g. [25]). Therefore, local properties of manifolds with the Killing property could be obtained from the study of complete (simply connected) Riemannian manifolds.

Proposition 9. Every simply connected complete Riemannian manifold $(M, g)$ with the local Killing property is a symmetric space and has the global Killing property. Every simply connected complete Riemannian manifold $(M, g)$ with the global Killing property is strongly Clifford-Wolf homogeneous.

Proof. Since $(M, g)$ is locally symmetric (by Proposition 8) complete and simply connected, then it is a symmetric space (see e.g. [25]). In particular, $(M, g)$ is really analytic. For any point $x$ in a given manifold $(M, g)$ there is a Killing frame $\left\{X_{1}, \ldots, X_{n}\right\}$ in some neighborhood $U$ of the point $x$. We state now that the above (local) Killing frame is uniquely extends to a global Killing frame in $(M, g)$. In fact, every locally defined Killing vector field on $(M, g)$ is a restriction of a globally defined Killing vector field (for instance, by Theorems 1 and 2 in [32], each local Killing vector field in any simply connected really analytic Riemannian manifold admits a unique extension to a Killing vector field on the whole manifold).

Let us prove the second assertion. By assumptions there is a global Killing frame $\left\{X_{1}, \ldots, X_{n}\right\}$ on $(M, g)$. This means that $g\left(X_{i}, X_{j}\right)=\delta_{i j}$ for every $1 \leq i, j \leq n$. Therefore, any linear combination of vector fields $X_{1}, \ldots, X_{n}$ over $\mathbb{R}$ is a Killing vector field of constant length. By completeness and Theorem 7 , we get that $(M, g)$ is restrictively CliffordWolf homogeneous. Now it is enough to apply the first assertion of the proposition and Theorem 9 .

q.e.d.

The authors of [15] tried but failed to classify simply connected complete Riemannian manifolds with the Killing property (see the page 407 right before the section 5 in [15]). The following theorem solves this question completely.

Theorem 11. A simply connected complete Riemannian manifold $(M, g)$ has the Killing property if and only if it is isometric to a direct metric product of Euclidean space, compact simply connected simple Lie groups with bi-invariant metrics, and round spheres $S^{7}$ (some mentioned factors could be absent). 
Remark 3. The assertion of Theorem 11 also follows from the first assertion of Proposition 9 and Theorem 9.1 in [43].

Proof. The sufficiency of this statement follows from the well-known fact that any mentioned factor has the Killing property (see also the next section as far as round $S^{7}$ is concerned).

Let us prove the necessity. As a corollary of Proposition 9 and Theorem $1,(M, g)$ must have a form as in Theorem 1 . But we may leave only $S^{7}$ as factors among odd-dimensional spheres by the following reason. By Theorem 4.1 in [15], every factor of the corresponding product also has the Killing property. It is well known (see also Corollary 2 below) that only $S^{3}$ and $S^{7}$ have the Killing property among (simply connected) odd-dimensional round spheres. But round $S^{3}$ can be considered as compact simply connected simple Lie group $S U(2)$ supplied with a bi-invariant Riemannian metric. So we can omit also $S^{3}$. q.e.d.

\section{Results of A. Hurwitz and J. Radon}

A. Hurwitz has posed the following question.

Question 2. For a given natural number $m$, to find the maximal natural number $p=\rho(m)$ such that there is a bilinear real vectorfunction $z=\left(z_{1}, \ldots, z_{m}\right)=z(x, y)$ of real vectors $x=\left(x_{1}, \ldots, x_{p}\right)$ and $y=\left(y_{1}, \ldots, y_{m}\right)$, which satisfies the equation

$$
\left(x_{1}^{2}+\cdots+x_{p}^{2}\right)\left(y_{1}^{2}+\cdots+y_{m}^{2}\right)=z_{1}^{2}+\cdots+z_{m}^{2} .
$$

for all $x \in \mathbb{R}^{p}, y \in \mathbb{R}^{m}$.

Using some equivalent formulations, J. Radon in [35] and A. Hurwitz in $[\mathbf{2 6}]$ independently obtained the following answer.

Theorem 12. If $m=2^{4 \alpha+\beta} m^{\prime}$, where $\beta=0,1,2,3, \alpha$ is a nonnegative integer, and $m^{\prime}$ is odd, then $\rho(m)=8 \alpha+2^{\beta}$.

Let us give some equivalent formulations and corollaries, following in some respect to J. Radon. It is clear that any bilinear function $z=z(x, y)$ can be represented in the form

$$
z=z(x, y)=\sum_{j=1}^{p} x_{j}\left(A_{j} y\right)=\left(\sum_{j=1}^{p} x_{j} A_{j}\right) y,
$$

where $A_{j}$ are $(m \times m)$-matrices and one considers $z$ and $y$ as vectorscolumns. Putting $x_{i}=\delta_{j i}, i=1, \ldots, p$ for a fixed $j$ from $\{1, \ldots, p\}$ and using equation (1), one can easily see that

1) every $A_{j}$ must be an orthogonal matrix.

On the other side, the same equation shows that

2 ) for any fixed $y \in \mathbb{R}^{m}, A_{j} y, j=1, \ldots, p$ must be mutually orthogonal vectors in $\mathbb{R}^{m}$ with lengths, all equal to $|y|$. 
As a corollary, we must have $p \leq m$. At the end, the equation (1) and the last form of equation (2) show that

3 ) for any unit vector $x \in \mathbb{R}^{p}$, the matrix $\left(\sum_{j=1}^{p} x_{j} A_{j}\right)$ must be orthogonal.

The last statement explains the title of the paper [35] and gives an equivalent form of the question 2 , which considered J. Radon.

Now, it is clear that if we change every matrix $A_{j}$ in the bilinear form (2) by $B_{j}=A_{j} A$, where $A$ is a fixed orthogonal $(m \times m)$-matrix, then we get another bilinear form, which also satisfies the equation (1). If we take $A=A_{p}^{-1}$, and denote $B_{j}$ one more by $A_{j}$, we get the following form of (2):

$$
z=z(x, y)=\sum_{j=1}^{p-1} x_{j}\left(A_{j} y\right)+x_{p}(I y)=\left(\sum_{j=1}^{p-1} x_{j} A_{j}+x_{p} I\right) y .
$$

Now, applying the properties 1) and 2) for new bilinear form (3), we get that all matrices $A_{j}, j=1, \ldots p-1$, in (3) must be both orthogonal and skew-symmetric (since $A_{j} y \perp y=I y$ for all $y \in \mathbb{R}^{m}$ ) (it implies in particular that $p \geq 2$ is possible only when $m$ is even). Now from Theorem 12, 1), 2) and the last statement it easily follows

Theorem 13. Any nontrivial collection of vector fields on $S^{m-1}$ consists of mutually orthogonal unit Killing vector field on $S^{m-1}$ if and only if it can be presented in a form $X_{j}(y)=A_{j} y, y \in S^{m-1}$, $j=1, \ldots, p-1$, where $(m \times m)$-matrices $A_{j}$, which are both orthogonal and skew-symmetric (thus $m$ is even), are taken from the equation (3), defining a bilinear form satisfying the equation (1). The maximal number of such fields is equal to $\rho(m)-1$, see Theorem 12 .

Theorem 13 implies

Theorem 14. A maximal dimension l of Clifford-Killing spaces $C K_{l}$ on $S^{m-1}$ is equal to $\rho(m)-1$.

Corollary 2. A maximal dimension l of Clifford-Killing spaces $C K_{l}$ on $S^{m-1}$ is equal to $m-1>0$ if and only if $m \in\{2,4,8\}$. As a corollary, $S^{1}, S^{3}$, and $S^{7}$ are all round spheres with the Killing property.

Note that the last result is related to the existence of algebras of complex, quaternion, and the Caley numbers.

One should note also that J.F. Adams has proved that there is no $\rho(m)$ continuous orthonormal (or, equivalently, linear independent) tangent vector fields on the sphere $S^{m-1}$ [1] (see also Theorem 13.10 of Chapter 15 in $[\mathbf{2 7}])$.

Later on, Eckmann reproved the Hurwitz-Radon Theorem in [19]. The methods of Radon and Hurwitz yield complicated schemes for actually constructing the forms $(3)$ for $p=\rho(m)$, which have been simplified 
by Adams, Lax, and Phillips [2], as well as by Zvengrowski [45] and by Balabaev [3].

\section{Clifford-Killing spaces on spheres and Clifford algebras and modules}

In this section we continue the study of Clifford-Killing spaces on spheres $S^{m-1}$, supplied with their canonical Riemannian metrics of constant sectional curvature 1. As noted before, those spaces are CliffordWolf homogeneous when $m$ is even. The material of Sections 9 through 11 is closely related to that of $[\mathbf{4 0}]$ and $[\mathbf{4 1}]$, especially in connection with the Hurwitz equations and Helgason spheres.

Theorem 15. Real $(m \times m)$-matrices $u_{1}, u_{2}, \ldots, u_{l}, l \geq 1$, define pairwise orthogonal at every point of $S^{m-1}$ unit Killing vector fields $U_{i}(x):=u_{i} x, x \in S^{m-1}$, on $S^{m-1}$ if and only if

$$
u_{i} \in O(m) \cap s o(m), \quad u_{i}^{2}=-I, \quad i=1, \ldots, l,
$$

and

$$
u_{i} u_{j}+u_{j} u_{i}=0, \quad i \neq j .
$$

In this case $m$ is even and $u_{i} \in S O(m)$.

Proof. The vector fields $U_{i}$ are unit Killing vector fields on $S^{m-1}$ if and only if they can be presented as follows: $U_{i}(x)=u_{i} x, \quad x \in$ $S^{m-1}$, where $u_{i} \in O(m) \cap s o(m)$. This implies that $m$ is even, $(x, y)=$ $\left(u_{i} x, u_{i} y\right)=-\left(u_{i}^{2} x, y\right)$, and $u_{i}^{2}=-I$ (here $(\cdot, \cdot)$ means the standard inner product in $\mathbb{R}^{m}$ ).

It is clear that under the first condition in (4), vector fields $U_{i}$ and $U_{j}$ on $S^{m-1}$ are orthogonal if and only if

$$
\left(\left(u_{i}+u_{j}\right) x,\left(u_{i}+u_{j}\right) x\right)=\left(u_{i} x, u_{i} x\right)+\left(u_{j} x, u_{j} x\right), \quad x \in S^{m-1},
$$

which is equivalent to the identity $\left(u_{i} u_{j} x, x\right) \equiv 0, x \in \mathbb{R}^{m}$, or $u_{i} u_{j} \in$ so $(m)$, or

$$
-\left(u_{i} u_{j}\right)=\left(u_{i} u_{j}\right)^{t}=u_{j}^{t} u_{i}^{t}=\left(-u_{j}\right)\left(-u_{i}\right)=u_{j} u_{i},
$$

i.e. (5).

A matrix $u_{i} \in O(m)$ is skew symmetric if and only if $u_{i}$ is orthogonally similar to a matrix $u=\operatorname{diag}(C, \ldots, C)$, where $C \in O(2)$ is skew symmetric. This implies that $u_{i} \in S O(m)$.

q.e.d.

Remark 4. According to Theorem 15, later on we may assume that $m$ is even and $m=2 n$.

Theorem 15 naturally leads to the notion of associative algebras over the field $\mathbb{R}$, with generators $e_{1}, \ldots, e_{l}$, such that $e_{i}^{2}=-1, e_{i} e_{j}+e_{j} e_{i}=$ $0, i \neq j$ (and any other relation in the algebra is some corollary of 
the indicated relations). Such algebra $C l_{l}$ is called the Clifford algebra (with respect to negatively definite quadratic form $-(y, y)$ in $\mathbb{R}^{k}$ and orthonormal basis $\left\{e_{1}, \ldots, e_{l}\right\}$ in $\left.\left(\mathbb{R}^{k},(\cdot, \cdot)\right)\right)$. These algebras include the algebra $C l_{1}=\mathbb{C}$ of complex numbers and the algebra $C l_{2}=\mathbb{H}$ of quaternions.

The others Clifford algebras can be described as follows: $C l_{3}=\mathbb{H} \oplus \mathbb{H}$, $C l_{4}=\mathbb{H} \otimes_{\mathbb{R}} \mathbb{R}(2)$, where the algebra $\mathbb{R}(2)$ is generated by symmetric $(2 \times 2)$-matrices $\operatorname{diag}\{1,-1\}$ and the permutation matrix of vectors in canonical basis $\left\{e_{1}, e_{2}\right\}$ for $\mathbb{R}^{2}$. After this one can apply "the periodicity law" $C l_{k+4}=C l_{k} \otimes_{\mathbb{R}} C l_{4}$. See more details in $[\mathbf{2 7}, \mathbf{2 1}]$.

Let us consider $L_{l, 2 n}$, the algebra of linear operators (on $\mathbb{R}^{2 n}$ ) that is generated by the operators (the matrices) $u_{i}, i=1, \ldots, l \geq 1$. Theorem 15 implies that $L_{l, 2 n}$ is a homomorphic image of the Clifford algebra $C l_{l}$. It is easy to see that the kernel of the natural homomorphism $c: C l_{l} \rightarrow L_{l, 2 n}$ is a two-sided ideal of $C l_{l}$. The above description of Clifford algebras shows that $C l_{l}, l \neq 4 k+3$, contains no proper two-sided ideal, while the Clifford algebra $C l_{l}=C l_{l-1} \oplus C l_{l-1}$ contains exactly two proper two-sided ideals $A_{1}$ and $A_{2}$ that are both isomorphic to $C l_{l-1}$ if $l=4 k+3$. Thus, in any case $L_{l, 2 n}$ is isomorphic either to $C l_{l}$, or to $C l_{l-1}$ (if $l \neq 4 k+3$, then necessarily $L_{l, 2 n}$ is isomorphic to $\left.C l_{l}\right)$.

Now we consider an example, where $L_{l, 2 n}$ is not isomorphic to $C l_{l}$.

Example 3. Let us consider a Lie algebra $C K_{3} \subset s o(2 n)$, where $2 n=4 k$. In this case $C K_{3}$ is the linear span of vectors $u_{1}, u_{2}, u_{3}:=$ $u_{1} u_{2}$. Clearly, the algebra $L_{3,2 n}$ is not isomorphic to $C l_{3}$. It is easy to calculate the dimensions of both these associative algebras. The dimension of $C l_{m}$ considered as a vector space over $\mathbb{R}$, is $2^{m}[\mathbf{2 7}]$. In particular, $\operatorname{dim}\left(C l_{3}\right)=8$. On the other hand, $L_{3,2 n}=\operatorname{Lin}\left\{1, u_{1}, u_{2}, u_{3}\right\}$, $\operatorname{dim}\left(L_{3,2 n}\right)=4$, because

$$
u_{1} u_{3}=u_{1} u_{1} u_{2}=-u_{2}, \quad u_{2} u_{3}=u_{2} u_{1} u_{2}=u_{1} .
$$

Notice that in this case $L_{3,2 n}$ is isomorphic to the quaternion algebra $\mathbb{H}=C l_{2}$.

The above mentioned homomorphism $c: C l_{l} \rightarrow L_{l, 2 n}$ defines a representation of Clifford algebra $C l_{l}$ in $\mathbb{R}^{2 n}$, so the last vector space together with the representation $c$ becomes a Clifford module (over $C l_{l}$ ). We saw that any Clifford-Killing space $C K_{l} \subset s o(2 n)$ defines the structure of Clifford module on $\mathbb{R}^{2 n}$ over $C l_{l}$. It is very important that in this way one can get arbitrary Clifford module.

We need some information on the classification of Clifford modules over $C l_{l}[\mathbf{2 7}]$ :

a) If $l \neq 4 k+3$, then there exists (up to equivalence) precisely one irreducible Clifford module $\mu_{l}$ over $C l_{l}$ with the representation $c_{l}$. Every Clifford module $\nu_{l}$ over $C l_{l}$ is isomorphic to the $m$-fold direct sum of $\mu_{l}$, 
that is,

$$
\nu_{l} \cong \oplus^{m} \mu_{l}
$$

b) If $l=4 k+3$, then there exist (up to equivalence) precisely two non-equivalent irreducible Clifford modules $\mu_{l, 1}, \mu_{l, 2}$ over $C l_{l}$ with representations $c_{1}=c_{l-1} \circ \pi_{1}$ and $c_{2}=c_{l-1} \circ \pi_{2}$, where $\pi_{i}$ is natural projection of $C l_{l}$ onto the ideal $A_{i}, i=1,2$. The modules $\mu_{1}, \mu_{2}$ have the same dimension and every Clifford module $\nu_{l}$ over $C l_{l}$ is isomorphic to

$$
\nu_{l} \cong \oplus^{m_{1}} \mu_{l, 1} \oplus \oplus^{m_{2}} \mu_{l, 2},
$$

for some non-negative integers $m_{1}, m_{2}$. It is clear that the representation of $C l_{l}$ corresponding to the module $\nu_{l}$ is exact if and only if both numbers $m_{1}$ and $m_{2}$ are non-zero.

The dimension $n_{0}$ of $\mu_{l}$, or $\mu_{l, 1}, \mu_{l, 2}$ is equal to $n_{0}=2^{4 \alpha+\beta}$, if $8 \alpha+$ $2^{\beta-1}-1<l \leq 8 \alpha+2^{\beta}-1$, where $\alpha$ is a non-negative integer and $\beta=0,1,2,3$. In some sense, this is the function, inverse to the RadonHurwitz function $\rho(m)$ from Theorem 12.

Previous discussion implies

Theorem 16. The sphere $S^{2 n-1}$ admits a Clifford-Killing space $C K_{l}$ if and only if $1 \leq l \leq \rho(2 n)-1$. In this case $n_{0}=n_{0}(l)$ divides 2n. All Clifford-Killing spaces $C K_{l}$ for $S^{2 n-1}$ are pairwise equivalent (all spaces $C K_{l} \subset$ so $(2 n)$ are equivalent with respect to $O(2 n)$ ) if and only if $l \neq 4 k+3$. If $l=4 k+3$, then there exist exactly $\left[n / n_{0}(l)\right]+$ 1 non-equivalent classes of Clifford-Killing spaces $C K_{l}$ for $S^{2 n-1}$. In particular, all Clifford-Killing spaces $C K_{\rho(2 n)-1}$ for $S^{2 n-1}$ are pairwise equivalent if and only if $2 n=2^{4 \alpha+\beta} n^{\prime}$, where $\alpha$ is a non-negative integer, $n^{\prime}$ is odd, and $\beta=1$ or $\beta=3$. If $\beta=0$ or $\beta=2$ above, then there exist exactly $\left[n^{\prime} / 2\right]+1$ non-equivalent classes of Clifford-Killing spaces $C K_{\rho(2 n)-1}$ for $S^{2 n-1}$.

This theorem together with Theorem 17 below give an exact number of (proper) equivalence classes for Clifford-Killing spaces on $S^{2 n-1}$.

Proposition 10. The set of unit Killing vector fields on the sphere $S^{2 n-1}$ represents by itself a union of two disjoint orbits with respect to the adjoint action of the group $S O(2 n)$, and one orbit with respect to the adjoint action of the group $O(2 n)$.

Proof. By Theorem 15, an arbitrary unit Killing vector field on the sphere $S^{2 n-1}$ is defined by a matrix $U$ from $S O(2 n) \cap s o(2 n)$ with the condition $U^{2}=-I$. Thus there is a matrix $A(U) \in O(2 n)$ such that $A(U) U A(U)^{-1}=\operatorname{diag}(C, \ldots, C)$, where $C$ is a fixed matrix $C \in S O(2) \cap$ so(2). Moreover, if $A^{\prime}(U)$ is another such matrix, then $A(U)\left[A^{\prime}(U)\right]^{-1} \in$ $S O(2 n)$. This implies that every two matrices $U, V \in S O(2 n) \cap s o(2 n)$ are equivalent in $O(2 n)$, and equivalent in $S O(2 n)$ if and only if $A(U) A(V)^{-1} \in S O(2 n)$. This finishes the proof.

q.e.d. 
Theorem 17. If $2 n \equiv 2(\bmod 4)$, then any two spaces of the type $C K_{l}$ (necessarily $l=1)$ are $S O(2 n)$-equivalent. If $2 n \equiv 0(\bmod 4)$, then any $O(2 n)$-equivalence class of spaces $C K_{l} \subset$ so $(2 n)$ contains exactly two $S O(2 n)$-equivalence classes.

Proof. We shall use the vectors $U$ and $V$ as in Proposition 10, where $A(U) \in S O(2 n)$ and $A(V) \in O(2 n) \backslash S O(2 n)$. Let suppose that $2 n \equiv$ $2(\bmod 4)$. Then $l=1$ and any space $C K_{1}$ is spanned onto some unit Killing vector field $X$. Note that $X$ is equivalent with $-X \in C K_{1}$ by means an orthogonal matrix with determinant -1 , thus either $X$ or $-X$ is equivalent to the vector $U$ in $S O(2 n)$.

Let suppose now that $2 n \equiv 0(\bmod 4)$. Consider any space $C K_{l}$, which is spanned onto unit Killing vector fields $U_{1}, \ldots, U_{l}$. If $l=1$, then $X_{1}$ is obviously equivalent to $-X_{1}$ in $S O(2 n)$. If $l>1$, then any of two unit Killing vector fields in $C K_{l}$ can be continuously deformed (in the set $C K_{l}$ ) to another one. So all unit Killing vector fields in $C K_{l}$ are simultaneously equivalent to (only one of) $U$ or $V$ in $S O(2 n)$, for example, to $U$.

Let's consider now $B \in O(2 n) \backslash S O(2 n)$ and the space $C K_{l}^{\prime}$, which is equivalent to $C K_{l}$ by $B$. Since nor $U$, neither $-U$ is equivalent to $V$ in $S O(2 n)$, then any unit Killing vector in $C K_{l}^{\prime}$ is equivalent to the vector $V$, which is not equivalent in $S O(2 n)$ to unit Killing vectors in $C K_{l}$. Thus spaces $C K_{l}$ and $C K_{l}^{\prime}$ are not equivalent to each other in $S O(2 n)$. On the other hand, since $S O(2 n)$ is an index 2 subgroup of $O(2 n)$, it is clear that any $O(2 n)$-equivalence class contains at most two $S O(2 n)$-equivalence classes.

q.e.d.

It should be noted that subspaces $C K_{l}$ of Lie algebras $s o(2 n)$ play an important role in various mathematical theories. For example, Theorem 16 and statements on the page 23 in [9] imply that there is a bijection between $O(2 n)$-classes of $C K_{l} \subset s o(2 n)$ (for all possible pairs $(2 n, l)$ of this type) and isometry classes of generalized Heisenberg groups studied at first by A. Kaplan in [28]. These are special two-step nilpotent groups admitting a one dimensional solvable Einstein extensions that are wellknown Damek-Ricci spaces [14]. Note that Damek-Ricci spaces are harmonic Riemannian manifolds and most of them are not symmetric. We refer the reader to $[\mathbf{9}, \mathbf{1 8}, \mathbf{2 2}, \mathbf{4}, \mathbf{1 3}, \mathbf{2 3}]$ and references therein for a deep theory of generalized Heisenberg groups and Damek-Ricci spaces. Note also that there are some useful generalizations of subspaces of the type $C K_{l}$ in Lie algebras $s o(2 n)$. One of them is a notion of uniform subspaces of $s o(2 n)[\mathbf{2 2}]$. Such subspaces are used for producing new Einstein solvmanifolds with two-step nilpotent nilradical (see [22] and [29] for details). 


\section{Clifford-Killing spaces for $S^{2 n-1}$ and Radon's unit spheres in $O(2 n)$}

Now we supply the Lie algebra $s o(2 n)$ with the following $\operatorname{Ad}(S O(2 n))$ invariant inner product:

$$
(U, V)=-\frac{1}{2 n} \operatorname{trace}(U V) .
$$

The Lie group $S O(2 n)$ supplied with the corresponding bi-invariant inner Riemannian metric $\rho$, is a symmetric space. This metric is uniquely extended to bi-invariant "metric" $\rho$ on $O(2 n)(\rho(x, y)=+\infty$ if and only if $x, y$ lie in different connected components).

Note that $(X, X)=1$ for every unit Killing field $X$ on the sphere $S^{2 n-1}$ supplied with the canonical metric of constant curvature 1, and the Killing form $B$ of $s o(2 n)$ is connected with the form (8) by the formula

$$
B(U, V)=2(n-1) \operatorname{trace}(U V)=-4 n(n-1)(U, V) .
$$

Let us remind that forms trace $(U V)$ and $B(U, V)$ on $s o(2 n)$ are forms associated with the identical and the adjoint representations of $s o(2 n)$ respectively $[\mathbf{1 6}]$.

Definition 7. Let $A_{1}, \ldots, A_{p}, 1 \leq p \leq \rho(2 n)$ are matrices from $O(2 n)$, defining a bilinear form (2). Then the set of all matrices of the form $\sum_{i=1}^{p} x_{i} A_{i}$, where $x=\left(x_{1}, \ldots, x_{p}\right)$ is a unit vector in $\mathbb{R}^{p}$, we will call Radon's unit sphere for the form (2) or simply Radon's unit sphere, and denote by $R S^{p-1 \mid 2 n}$.

It follows from Section 8 and Theorem 15 that always $R S^{p-1 \mid 2 n} \in$ $O(2 n)$, and $R S^{p-1 \mid 2 n} \in S O(2 n)$ (respectively $R S^{p-1 \mid 2 n} \notin S O(2 n)$ ) if and only if at least one of matrices $A_{1}, \ldots, A_{p}$ is in $S O(2 n)$ (is not in $S O(2 n))$. Moreover, $R S^{p-1 \mid 2 n} A$ is also Radon's unit sphere for every $A \in O(2 n)$, and left and right translations are isometries in $(S O(2 n), \rho)$. Thus, from geometric viewpoint, we can consider only the Radon unit sphere, defined by a form (3). As it was said in Section 8 , in this case $A_{p}=I$, and the linear span of $A_{1}, \ldots, A_{p-1}$ is a Clifford-Killing space $C K_{l} \subset s o(2 n)$, where $l=p-1$. The goal of this section is the following

Theorem 18. In the notation of Definition 7, the map

$$
x=\left(x_{1}, \ldots, x_{l+1=p}\right) \in S^{l} \subset \mathbb{R}^{l+1} \rightarrow \sum_{j=1}^{p} x_{j}\left(A_{j}\right) \in(O(2 n), \rho)
$$

preserves distances and has the image $R S^{l \mid 2 n}$. As a corollary, any Radon's sphere $R S^{l \mid 2 n}$, defined by a form (2), is a totally geodesic submanifold in $(O(2 n), \rho)$, which is isometric to the standard unit sphere $S^{l} \subset \mathbb{R}^{l+1}$ with unit sectional curvature. If $A_{p}=I$, it is equal to $\exp \left(C K_{l}\right)$, where $C K_{l}$ is the linear span of $A_{1}, \ldots, A_{l=p-1}$ from the 
formula (3). Conversely, the image $\exp \left(C K_{l}\right) \subset S O(2 n)$ of arbitrary Clifford-Killing space $C K_{l} \subset$ so $(2 n)$ is a Radon's unit sphere $R S^{l \mid 2 n}$ for some form (3).

Proof. At first we shall prove the third assertion. It was proved in Section 8 and Theorem 15 that all $A_{1}, \ldots, A_{p-1=l}$ are elements of $S O(2 n) \cap$ $s o(2 n)$, which defines mutually orthogonal unit Killing vector fields on $S^{2 n-1}$. Let $C \in R S^{l \mid 2 n} \subset S O(2 n)$, that is $C=\sum_{i=1}^{l} x_{i} A_{i}+x_{l+1} I$, where $x=\left(x_{1}, \ldots, x_{l+1}\right)$ is a unit vector in $\mathbb{R}^{l+1}$. Let suppose at first that $x_{l+1}^{2} \neq 1$. Then the matrix

$$
A:=\frac{1}{\sqrt{\sum_{i=1}^{l} x_{i}^{2}}} \sum_{i=1}^{l} x_{i} A_{i}
$$

is in $S O(2 n) \cap s o(2 n) \cap C K_{l}$ and defines a unit Killing vector field on $S^{2 n-1}$. Obviously the vector $C$ can be represented in a form $C=$ $(\cos r) I+(\sin r) A, r \in \mathbb{R}$, where $\cos r=x_{l+1}$. Now for any $t, s \in \mathbb{R}$ we have $A^{2}=-I$ by Theorem 15 and so

$$
[(\cos t) I+(\sin t) A][(\cos s) I+(\sin s) A]=(\cos (t+s)) I+(\sin (t+s)) A .
$$

This means that the set of matrices $(\cos t) I+(\sin t) A, t \in \mathbb{R}$, constitutes a one-parameter subgroup in $S O(2 n)$ with the tangent vector $A$, and $C=\exp (r A) \in \exp \left(C K_{l}\right)$. If $x_{l+1}=1$ or $x_{l+1}=-1$, then for any matrix $A \in C K_{l}$ defining a unit Killing vector field, we have by the above arguments that $\exp (t A) \in R S^{l \mid 2 n}$ for all $t \in \mathbb{R}$, and $\exp (0 A)=C$ or $\exp (\pi A)=C$. So, we have proved the required equality $R S^{l \mid 2 n}=$ $\exp \left(C K_{l}\right)$. This implies in particular that $\exp \left(C K_{l}\right)$ is topologically $S^{l}$.

By Definition 7, any subspace $C K_{l=p-1}$ on $S^{2 n-1}$ has a basis of $l$ mutually orthogonal unit Killing vector fields, which are defined by some matrices $A_{1}, \ldots A_{p-1}$ in $S O(2 n) \cap s o(2 n)$ by Theorem 15. (Let us note also that by the same Theorem, $A_{i} A_{j}=-A_{j} A_{i}$, if $i \neq j$, and so $\left(A_{i}, A_{j}\right)=0$ by the formula (8).) It is clear now that the formula (3) defines a required bilinear form, and the last assertion follows from the third one.

By the last two assertions, $R S^{l \mid 2 n}=\exp C K_{l}$, where $C K_{l}$ is the linear span of $A_{1}, \ldots, A_{l}$, where $l=p-1$. Now, if $A \in C K_{l} \cap S O(2 n)$, then by Theorem $15, A^{2}=-I$ and the formula (8) gives us that $(A, A)=1$. This fact, the $\operatorname{Ad}(S O(2 n))$-invariance of the inner product (8), and the discussions in the first part of the proof implies that $\exp (t A), t \in \mathbb{R}$, is a geodesic circle in $S O(2 n)$ of the length $2 \pi$, entirely lying in $R S^{l \mid 2 n}$.

Let suppose that $B$ and $C$ are two different points in $R S^{l \mid 2 n}$, defined by unit vectors $b$ and $c$ in $\mathbb{R}^{l+1}$. Then there is a unit vector $d \in \mathbb{R}^{l+1}$, which is orthogonal to $b$, such that $c=(\cos r) b+(\sin r) d$ for some $r \in[0, \pi]$. The vector $d$ defines the corresponding element $D \in R S^{l \mid 2 n}$. 
Matrices $A_{1}^{\prime}=B, A_{2=p}^{\prime}=D$ define a bilinear form $z(x, y)$ with necessary properties by the formula (2), and corresponding Radon's sphere $R S^{1,2 n} \subset O(2 n)$, containing the points $A, D, C$. By the previous results, the right translation by $D^{-1}$, which is an isometry on $(O(2 n), \rho)$, transforms this Radon's sphere to another one of the form $\exp \left(C K_{1}\right)$, which is a geodesic circle in $(S O(2 n), \rho)$ of the length $2 \pi$. This implies that the curve $c(t):=(\cos t) B+(\sin t) D, t \in[O, r]$, joining the points $B$ and $C$ in $R S^{k \mid 2 n}$, is a shortest geodesic in $(O(2 n), \rho)$, parameterized by the arc-length. Thus we have proved the second assertion. The first assertion follows from the second one and the bi-invariance of the "metric" $\rho$ on $O(2 n)$.

q.e.d.

\section{Lie triple systems in $s o(2 n)$ and totally geodesic spheres in $S O(2 n)$}

Recall that a linear subspace $\mathfrak{a}$ of a Lie algebra $\mathfrak{g}$ is called Lie triple system if $[\mathfrak{a},[\mathfrak{a}, \mathfrak{a}]] \subset \mathfrak{a}$. We shall need the following

Lemma 6. If $\mathfrak{a}$ is a Lie triple system of a Lie algebra $\mathfrak{g}$, then $\mathfrak{h}:=$ $[\mathfrak{a}, \mathfrak{a}]$ and $\mathfrak{k}:=\mathfrak{h}+\mathfrak{a}$ are subalgebras of $\mathfrak{g}, \mathfrak{h} \cap \mathfrak{a}$ is an ideal of $\mathfrak{k}$.

Proof. From $[\mathfrak{a},[\mathfrak{a}, \mathfrak{a}]] \subset \mathfrak{a}$ and the Jacobi identity we get $[\mathfrak{h}, \mathfrak{h}] \subset \mathfrak{h}$ and $[\mathfrak{h}, \mathfrak{a}] \subset \mathfrak{a}$, that proves the first assertion of the lemma. Now, it is easy to see that $[\mathfrak{a}, \mathfrak{h} \cap \mathfrak{a}] \subset \mathfrak{h} \cap \mathfrak{a}$ and $[\mathfrak{h}, \mathfrak{h} \cap \mathfrak{a}] \subset \mathfrak{h} \cap \mathfrak{a}$, which proves the second assertion.

q.e.d.

Lemma 7. Let $G$ be a compact Lie group supplied with a bi-invariant Riemannian metric $\rho$. Suppose that $\mathfrak{a}$ is a Lie triple system in $\mathfrak{g}$, the Lie algebra of $G$. Then $M:=\exp (\mathfrak{a})$ is a totally geodesic submanifold of $G$, in particular, $M$ is a symmetric space. If universal Riemannian covering of $M$ is irreducible, then one of the following conditions holds:

1) $\mathfrak{a}$ is a simple Lie subalgebra of $\mathfrak{g}$ and $M$ is a simple compact Lie group with a bi-invariant Riemannian metric.

2) The Lie algebra $\mathfrak{h}:=[\mathfrak{a}, \mathfrak{a}]$ satisfies the relation $\mathfrak{h} \cap \mathfrak{a}=0$, and $(\mathfrak{h} \oplus$ $\mathfrak{a}, \mathfrak{h})$ is a symmetric pair corresponding to $M$. In particular, $\exp (\mathfrak{h} \oplus \mathfrak{a})$ is the full connected isometry group of $M$. If in this case the Lie algebra $\mathfrak{h} \oplus \mathfrak{a}$ is not simple, then $M$ is a simple compact Lie group with a biinvariant Riemannian metric.

Proof. Here we consider $\exp (\mathfrak{a})$ and $\exp (\mathfrak{h} \oplus \mathfrak{a})$ in the Lie theoretical sense. On the other hand, since $\rho$ is bi-invariant, they could be treated also in the Riemannian sense. Let $(\cdot, \cdot)$ be a $\operatorname{Ad}(G)$-invariant inner product on $\mathfrak{g}$ that generates $\rho$.

Let us consider the standard representation of $(G, \rho)$ as a symmetric space: $G \times G / \operatorname{diag}(G)$. Consider $\widetilde{\mathfrak{g}}=\mathfrak{g} \oplus \mathfrak{g}$, the Lie algebra of $G \times G$, and

$$
\widetilde{\mathfrak{k}}=\operatorname{diag}(\mathfrak{g}) \subset \widetilde{\mathfrak{g}}, \quad \widetilde{\mathfrak{p}}=\{(X,-X) \mid X \in \mathfrak{g}\} .
$$


Then $\widetilde{\mathfrak{g}}=\widetilde{\mathfrak{k}} \oplus \widetilde{\mathfrak{p}}$ is a Cartan decomposition for the symmetric space $G \times G / \operatorname{diag}(G)$. Now consider $\widetilde{\mathfrak{a}}=\{(X,-X) \mid X \in \mathfrak{a}\} \subset \widetilde{\mathfrak{p}}$. It is clear that $\widetilde{\mathfrak{a}}$ is a Lie triple system in $\widetilde{\mathfrak{g}}$, $[\widetilde{\mathfrak{a}}, \widetilde{\mathfrak{a}}]=\mathfrak{h} \oplus \mathfrak{h} \subset \widetilde{\mathfrak{k}}$. Using the standard theory of Lie triple system in symmetric spaces (see e.g. [25]), we conclude that the pair $([\widetilde{\mathfrak{a}}, \mathfrak{\mathfrak { a }}] \oplus \widetilde{\mathfrak{a}}, \widetilde{\mathfrak{a}})$ is symmetric and corresponds to the symmetric space $M_{1}:=\exp (\widetilde{\mathfrak{a}})$. It is clear that $M_{1}$ (supplied with Riemannian metric induced by the inner product $\frac{1}{2}(\cdot, \cdot)+\frac{1}{2}(\cdot, \cdot)$ on $\left.\mathfrak{g} \oplus \mathfrak{g}\right)$ is isometric to $M$. In this case it is sufficient to consider an isometry $i: M \rightarrow M_{1}$ defined as follows: $i(\exp (t X))=(\exp (t X), \exp (-t X))$, where $t \in \mathbb{R}, X \in \mathfrak{a}$. This proves the first assertion of the lemma.

Let us suppose that the universal covering of $M=M_{1}$ is an irreducible symmetric space. Then the Lie algebra $[\widetilde{\mathfrak{a}}, \mathfrak{\mathfrak { a }}] \oplus \tilde{\mathfrak{a}}$, being the Lie algebra of the full isometry group of $M_{1}$, is either simple or a direct sum of two copies of some simple Lie algebra. Recall that $\mathfrak{u}:=\mathfrak{h} \cap \mathfrak{a}$ is an ideal in the Lie algebra $\mathfrak{h}+\mathfrak{a}$ by Lemma 6 . Let us consider maps

$$
\pi_{1}:[\widetilde{\mathfrak{a}}, \widetilde{\mathfrak{a}}] \oplus \widetilde{\mathfrak{a}} \rightarrow \mathfrak{h}+\mathfrak{a}, \quad \pi_{2}:[\tilde{\mathfrak{a}}, \tilde{\mathfrak{a}}] \oplus \widetilde{\mathfrak{a}} \rightarrow \mathfrak{h}+\mathfrak{a},
$$

defined as follows. Let $Z=(Y, Y)+(X,-X)$, where $X \in \mathfrak{a}$ and $Y \in$ $[\mathfrak{a}, \mathfrak{a}]$, then we put $\pi_{1}(Z)=Y+X$ and $\pi_{2}(Z)=Y-X$. It is easy to see that $\pi_{1}$ and $\pi_{2}$ are Lie algebra epimorphisms. The kernel of $\pi_{1}$ is $\mathfrak{u}_{1}=\{(0, X) \mid X \in \mathfrak{u}\}$ and the kernel of $\pi_{2}$ is $\mathfrak{u}_{2}=\{(X, 0) \mid X \in \mathfrak{u}\}$. In particular, we get that $\mathfrak{u} \oplus \mathfrak{u}=\mathfrak{u}_{1} \oplus \mathfrak{u}_{2}$ is an ideal in the Lie algebra $[\widetilde{\mathfrak{a}}, \tilde{\mathfrak{a}}] \oplus \widetilde{\mathfrak{a}}$.

Suppose that the Lie algebra $[\widetilde{\mathfrak{a}}, \widetilde{\mathfrak{a}}] \oplus \widetilde{\mathfrak{a}}$ is isomorphic to $\mathfrak{s} \oplus \mathfrak{s}$, where $\mathfrak{s}$ is a simple Lie algebra. If $\mathfrak{u}$ is not trivial, then $[\widetilde{\mathfrak{a}}, \tilde{\mathfrak{a}}] \oplus \widetilde{\mathfrak{a}}$ coincides with its ideal $\mathfrak{u} \oplus \mathfrak{u}=\mathfrak{u}_{1} \oplus \mathfrak{u}_{2}$ (this ideal could not be proper in this case). Moreover, $\mathfrak{u}=\mathfrak{a}=[\mathfrak{a}, \mathfrak{a}]=\mathfrak{h}$ is a simple Lie algebra (since $\mathfrak{u}_{1}$ is the kernel of $\pi_{1}$, then $\mathfrak{h}+\mathfrak{a}$ is isomorphic to $\left.\mathfrak{u}_{2} \sim \mathfrak{u}=\mathfrak{h} \cap \mathfrak{a}\right)$. In this case $M$ is a connected simple compact Lie group supplied with a bi-invariant Riemannian metric, and we get Condition 1 ) of the lemma. If $\mathfrak{u}$ is trivial, then $\pi_{1}$ is an isomorphism. Therefore, $\mathfrak{h}+\mathfrak{a}=\mathfrak{h} \oplus \mathfrak{a}$ is isomorphic to $\mathfrak{s} \oplus \mathfrak{s}$, and $\mathfrak{h}$ is isomorphic to $\operatorname{diag}(\mathfrak{s})$ ( $\operatorname{since} \operatorname{diag}(\mathfrak{s})$ is a unique proper subalgebra in $\mathfrak{s} \oplus \mathfrak{s}$ ). Hence $M$ is a simple compact Lie group (with the Lie algebra isomorphic to $\mathfrak{s}$ ) with a bi-invariant Riemannian metric, and we get Condition 2) of the lemma with non-simple $\mathfrak{h} \oplus \mathfrak{a}$.

Now, if the Lie algebra $[\widetilde{\mathfrak{a}}, \widetilde{\mathfrak{a}}] \oplus \widetilde{\mathfrak{a}}$ is simple, then $\mathfrak{u}$ is trivial and $\pi_{1}$ is an isomorphism. Therefore, $\mathfrak{h}+\mathfrak{a}=\mathfrak{h} \oplus \mathfrak{a}=\pi_{1}([\widetilde{\mathfrak{a}}, \widetilde{\mathfrak{a}}] \oplus \widetilde{\mathfrak{a}})$ is a simple Lie algebra that is the Lie algebra of the full isometry group of $M$. Moreover, by the definition of $\pi_{1}$ we obtain $\pi_{1}([\widetilde{\mathfrak{a}}, \widetilde{\mathfrak{a}}])=\mathfrak{h}$. Therefore, Condition 2) of the lemma with simple $\mathfrak{h} \oplus \mathfrak{a}$ holds.

Theorem 19. Let $C K_{l}$ be a Clifford-Killing subspace of dimension $l$ in the Lie algebra so $(2 n)$. Then the following statements are true:

1) $C K_{l}$ is a Lie triple system in the Lie algebra so $(2 n)$. 
2) For every $C K_{1}$ the image $\exp \left(C K_{1}\right)$ is a closed geodesic of length $2 \pi$ in $(S O(2 n), \rho)$. If $l \geq 2$, then the image $\exp \left(C K_{l}\right)$ is a totally geodesic sphere $S^{l}$ of constant sectional curvature 1 in $(S O(2 n), \rho)$.

3) If $C K_{l}$ is a Lie subalgebra of $s o(2 n)$ then either $l=1$, or $l=3$.

4) Every $C K_{1}$ is a commutative Lie subalgebra of so(2n), the image $\exp \left(C K_{1}\right)$ consist of Clifford-Wolf translations of $S^{2 n-1}$, and the $\exp \left(C K_{1}\right)$-orbits constitute a totally geodesic foliation of equidistant (great) circles in the sphere $S^{2 n-1}$.

5) If $\mathrm{CK}_{3}$ is a Lie subalgebra of so(2n) then $\mathrm{CK}_{3}=\left[\mathrm{CK}_{3}, C K_{3}\right]$ is isomorphic to so(3) $\sim s u(2)$, and $\exp \left(C K_{3}\right)=S^{3}$ is the group $S U(2)$ supplied with a bi-invariant Riemannian metric. Moreover, $\exp \left(C K_{3}\right)=$ $S U(2)$ consists of Clifford-Wolf translations of $S^{2 n-1}$ and, consequently, the $\exp \left(\mathrm{CK}_{3}\right)$-orbits constitute a totally geodesic foliation of equidistant (great) 3-spheres in the sphere $S^{2 n-1}$.

6) If $C K_{l}$ is not a Lie subalgebra of so(2n) then $C K_{l} \cap\left[C K_{l}, C K_{l}\right]$ is trivial, subspaces $C K_{l} \oplus\left[C K_{l}, C K_{l}\right]$ and $\left[C K_{l}, C K_{l}\right]$ are Lie subalgebras of so(2n) such that the pair $\left(C K_{l} \oplus\left[C K_{l}, C K_{l}\right],\left[C K_{l}, C K_{l}\right]\right)$ is the symmetric pair $(s o(l+1)$, so $(l))$ and $\exp \left(C K_{l}+\left[C K_{l}, C K_{l}\right]\right)$ is isomorphic to $S O(l+1)$, the full connected isometry group of $\exp \left(C K_{l}\right)=S^{l}$.

Remark 5. Some variants of this theorem are admissible in the literature (see e.g. $[\mathbf{1 7}]$ ).

Proof. Let $C K_{l}$ be the linear span of unit Killing vector fields $U_{1}, \ldots$, $U_{l}$. By Theorem 15 we get that $U_{i}^{2}=-I$ and $U_{i} U_{j}+U_{j} U_{i}=0$ for $i \neq j$. Let us show that $\left[C K_{l},\left[C K_{l}, C K_{l}\right]\right] \subset C K_{l}$. If the indices $i, j, k$ are pairwise distinct, then $\left[U_{i}, U_{j}\right]=2 U_{i} U_{j}$ and

$$
\left[U_{k},\left[U_{i}, U_{j}\right]\right]=2 U_{k} U_{i} U_{j}-2 U_{i} U_{j} U_{l}=-2 U_{i} U_{k} U_{j}+2 U_{i} U_{k} U_{j}=0 .
$$

On the other hand,

$$
\left[U_{i},\left[U_{i}, U_{j}\right]\right]=2 U_{i} U_{i} U_{j}-2 U_{i} U_{j} U_{i}=-2 U_{j}+2 U_{i} U_{i} U_{j}=-4 U_{j} \in C K_{l} .
$$

Therefore, $C K_{l}$ is a Lie triple system in $s o(2 n)$, that proves the first assertion of the theorem.

The second assertion immediately follows from Theorem 18 .

If $C K_{l}$ is a Lie algebra, then necessarily $l=1$ or $l=3$, that proves the third assertion of the theorem.

The fourth assertion immediately follows from Theorem 8 .

If $C K_{3}$ is a Lie subalgebra of $s o(2 n)$, then $C K_{3}$ is isomorphic to $s o(3) \sim s u(2)$. In this case $\exp \left(C K_{3}\right)=S U(2)=S^{3}$ with a metric of constant sectional curvature 1 . Obviously, every $X \in C K_{3}$ is a Killing vector field of constant length on $S^{2 n-1}$. By Theorem 8 , we get that $\exp \left(C K_{3}\right)=S U(2)$ consists of Clifford-Wolf translations of $S^{2 n-1}$. Now, it easy to see that $\exp \left(C K_{3}\right)$-orbits constitute a totally geodesic foliation of equidistant (great) 3 -spheres in the sphere $S^{2 n-1}$. This proves the fifth assertion of the theorem. 
By Lemma 7, $C K_{l}+\left[C K_{l}, C K_{l}\right]$ is a Lie subalgebra of $s o(2 n)$ and the subgroup $\exp \left(C K_{l}+\left[C K_{l}, C K_{l}\right]\right)$ of $S O(2 n)$ is a connected isometry group of $M:=\exp \left(C K_{l}\right)=S^{l}$. If $l=3$ and $C K_{3}$ is not a Lie subalgebra of $s o(2 n)$, then (by Lemma 7) the Lie algebra $C K_{3}+\left[C K_{3}, C K_{3}\right]=$ $C K_{3} \oplus\left[C K_{3}, C K_{3}\right]$ is isomorphic to $s o(4) \sim s o(3) \oplus s o(3)$ and $\exp \left(C K_{3} \oplus\right.$ $\left.\left[C K_{3}, C K_{3}\right]\right)=S O(4)$. Now, suppose that $l \neq 1,3$. Then the sphere $S^{l}=\exp \left(C K_{l}\right)$ is not a Lie subgroup of $S O(2 n)$. By Lemma 7, we get that $C K_{l} \cap\left[C K_{l}, C K_{l}\right]$ is trivial, the Lie algebra $C K_{l} \oplus\left[C K_{l}, C K_{l}\right]$ is $s o(l+1)$ and $\exp \left(C K_{l} \oplus\left[C K_{l}, C K_{l}\right]\right)=S O(l+1)$.

The theorem is completely proved.

q.e.d.

According to Theorems 18 and 19, the study of subspaces $C K_{l}$ is related to the study of totally geodesic spheres in $S O(2 n)$.

Note that there are well known totally geodesic spheres in $S O(2 n)$, the Helgason spheres. In the paper [24], S. Helgason proved that every compact irreducible Riemannian symmetric space $M$ with the maximal sectional curvature $\varkappa$ contains totally geodesic submanifolds of constant curvature $\varkappa$. Any two such submanifolds of the same dimension are equivalent under the full connected isometry group of $M$. The maximal dimension of any such submanifold is $1+m(\bar{\delta})$, where $m(\bar{\delta})$ is the multiplicity of the highest restricted root $\bar{\delta}$. Moreover, if $M$ is not a real projective space, then such submanifolds of dimension $1+m(\bar{\delta})$ are actually spheres.

In the case when $M$ is a simple compact Lie group with a bi-invariant Riemannian metric, $m(\bar{\delta})=2$. Therefore, the maximal dimension of submanifolds as above is 3 . Note that $M=(S O(2 n), \rho), n \geq 2$, is not a real projective space, therefore, there are 3-dimensional totally geodesic Helgason's spheres in $(S O(2 n), \rho)$. It is easy to give a description of these spheres (see [24]). At first we recall some well known facts (see e.g. $H$ of Chapter 8 in $[\mathbf{1 1}])$. Let $E_{i j}$ be a $(2 n \times 2 n)$-matrix with zero entries except the $(i, j)$-th entry that is 1 . Consider matrices $F_{i}=$ $E_{(2 i)(2 i-1)}-E_{(2 i-1)(2 i)}$ for $1 \leq i \leq n$. These matrices define a basis of a standard Cartan subalgebra $\mathfrak{k}$ in $s o(2 n)$. Hence every $X$ in $\mathfrak{k}$ has the form $X=\sum_{i=1}^{n} \lambda_{i} F_{i}$. Every root (with respect to $\mathfrak{k}$ ) of $s o(2 n)$ has the form $\lambda_{i} \pm \lambda_{j}, i \neq j$. Note that all these roots have the same length.

Let $V_{\lambda_{i} \pm \lambda_{j}}$ be the (two-dimensional) root space of the root $\lambda_{i} \pm \lambda_{j}$ in $s o(2 n)$. Put $U_{\lambda_{i} \pm \lambda_{j}}=\mathbb{R} \cdot\left(F_{i} \pm F_{j}\right) \oplus V_{\lambda_{i} \pm \lambda_{j}}$. In this notation, $\exp \left(U_{\lambda_{i} \pm \lambda_{j}}\right)$ is a Helgason's sphere in $(S O(2 n), \rho)$ (see details in the proof of Theorem 1.2 in [24]). Moreover, by Theorem 1.1 in [24] any two Helgason's spheres in $(S O(2 n), \rho)$ are equivalent under the full connected isometry group of $(S O(2 n), \rho)$. Therefore, every Helgason's sphere in $(S O(2 n), \rho)$ is conjugate in $S O(2 n)$ either to the sphere $\exp \left(U_{\lambda_{1}-\lambda_{2}}\right)$, or to the sphere $\exp \left(U_{\lambda_{1}+\lambda_{2}}\right)$. 
Proposition 11. The spheres $S^{3}=\exp \left(C K_{3}\right)$ in Assertion 5 of Theorem 19 are Helgason's spheres of constant curvature 1 in $(S O(4), \rho)$. Every Clifford-Killing space $\mathrm{CK}_{3}$ in so(4) is an ideal of so(4).

Proof. Note that $s o(4) \cong s o(3) \oplus s o(3)$. There are only two nonproportional roots for the standard Cartan algebra $\mathfrak{k}: \lambda_{1}+\lambda_{2}$ and $\lambda_{1}-\lambda_{2}$. It is easy to see that $U_{\lambda_{1}+\lambda_{2}}$ and $U_{\lambda_{1}-\lambda_{2}}$ are pairwise commuting Lie algebras isomorphic to $s o(3) \sim s u(2)$. In particular, $\exp \left(U_{\lambda_{1} \pm \lambda_{2}}\right)$ are spheres of the type $\exp \left(C K_{3}\right)$ in $(S O(4), \rho)$ (see Assertion 5 in Theorem 19). Note also, that $U_{\lambda_{1}+\lambda_{2}}$ and $U_{\lambda_{1}-\lambda_{2}}$ could be naturally identified with the Lie algebras of left and right shifts on $S^{3}=S U(2)$. On the other hand, (from the previous discussion) $\exp \left(U_{\lambda_{1} \pm \lambda_{2}}\right)$ are Helgason's spheres $(S O(4), \rho)$. By Assertion 2 in Theorem 19 (or by Theorem 18) these spheres have constant curvature 1 . This proves the first assertion of the proposition.

Now, let $C K_{3}$ be an arbitrary Clifford-Killing space of so(4). By Theorem 18, $\exp \left(C K_{3}\right)$ is a sphere $S^{3}$ of constant curvature 1. Since its sectional curvature coincides with the sectional curvature of Helgason's spheres $\exp \left(U_{\lambda_{1} \pm \lambda_{2}}\right)$, then $\exp \left(C K_{3}\right)$ should be a Helgason's sphere too. From the description of Helgason's spheres right before the statement of the proposition we get, that $\exp \left(C_{3}\right)$ is conjugate in $S O(4)$ either to $\exp \left(U_{\lambda_{1}-\lambda_{2}}\right)$, or to $\exp \left(U_{\lambda_{1}+\lambda_{2}}\right)$. In particular, $C K_{3}$ is conjugate in $S O$ (4) either to $U_{\lambda_{1}-\lambda_{2}}$, or to $U_{\lambda_{1}+\lambda_{2}}$. Since $U_{\lambda_{1} \pm \lambda_{2}}$ are ideals in $s o(4)$, this proves the second assertion of the proposition.

q.e.d.

Proposition 12. Every Helgason's sphere in $(S O(2 n), \rho), n \geq 2$, has the constant sectional curvature $n / 2$. In particular, for $n \geq 3$ all Helgason's spheres are distinct from the spheres in Theorem 18.

Proof. For $n=2$ the assertion of the proposition follows from Proposition 11 . Now, let us consider the case $n \geq 3$. The subgroup $H=$ $\operatorname{diag}(S O(4), 1, \ldots, 1) \subset S O(2 n)$ with a Riemannian bi-invariant metric $\rho_{1}$ induced by $\rho$, is a totally geodesic submanifold in $(S O(2 n), \rho)$. On the other hand, all roots of $\mathfrak{h}=\operatorname{Lie}(H)$ are roots of $s o(2 n)$. From the above description of the Helgason spheres we get that every Helgason's sphere in $\left(H, \rho_{1}\right)$ is also a Helgason's sphere in $(S O(2 n), \rho)$. It is easy to see that $\left(H, \frac{n}{2} \rho_{1}\right)$ is isometric to $\left(S O(4), \rho^{\prime}\right)$, where $\rho^{\prime}$ is a bi-invariant Riemannian metric, generated by the inner product (8) for $n=2$. Since all Helgason's spheres in $\left(S O(4), \rho^{\prime}\right)$ has constant curvature 1 by Proposition 11 , then every Helgason's sphere in $\left(H, \rho_{1}\right)$ has constant curvature $n / 2$.

q.e.d.

\section{Lie algebras in Clifford-Killing spaces on $S^{2 n-1}$}

Below we discuss some results related to Lie algebras containing in Clifford-Killing subspaces $C K_{l}$ of $s o(2 n)$. 
Proposition 13. Let $X, Y$ be linearly independent Killing vector fields on $S^{2 n-1}$ with constant inner products $\operatorname{can}(X, X)$, can $(Y, Y)$, $\operatorname{can}(X, Y)$. Then the Lie bracket $[X, Y]$ is a non-trivial Killing vector field of constant length on $S^{n-1}$. If $X, Y$ are unit mutually orthogonal Killing vector fields on $S^{2 n-1}$, then the triple of vector fields

$$
\left\{X, Y, Z:=\frac{1}{2}[X, Y]\right\}
$$

constitutes an orthonormal basis of Lie algebra $C K_{3}$ of vector fields on $S^{2 n-1}$ with relations $[X, Y]=2 Z,[Z, X]=2 Y,[Y, Z]=2 X$. Consequently, Assertion 5) of Theorem 19 holds.

Proof. By Corollary 1 we get $\frac{1}{2}[X, Y]=\nabla_{X} Y=-\nabla_{Y} X$, and by Proposition 1, the formula $\operatorname{can}\left(\nabla_{X} Y, \nabla_{X} Y\right)=\operatorname{can}(R(X, Y) Y, X)$ holds. Since $\operatorname{can}(X, Y)$ and the sectional curvature of $\left(S^{n-1}\right.$, can $)$ are constant, the expression $\operatorname{can}(R(X, Y) Y, X)$ is a positive constant. Consequently, $[X, Y]$ is a (non-trivial) Killing field of constant length, that proves the first assertion of the proposition.

The orthonormality of the triple of Killing vector fields $\{X, Y, Z\}$ on $S^{2 n-1}$ follows from the orthonormality of the pair $\{X, Y\}$, the proof of the first part of Proposition, and relations $0=Y \operatorname{can}(X, X)=$ $-\operatorname{can}([X, Y], X), 0=X \operatorname{can}(Y, Y)=\operatorname{can}([X, Y], Y)$. The first commutation relation follows from the definition. On the ground of Theorem 15, we get the equality:

$$
\begin{gathered}
{[Z, X]=\frac{1}{2}\{(X Y-Y X) X-X(X Y-Y X)\}=} \\
\frac{1}{2}\{X Y X-Y X X-X X Y+X Y X\}=\frac{1}{2}(2 Y+2 Y)=2 Y .
\end{gathered}
$$

The third commutation relation can be proved analogously. Then the linear span $C K_{3}$ of vectors $X, Y, Z$ is a Lie algebra. Now, it suffices to apply Theorem 19 .

q.e.d.

Proposition 14. If $l \geq 4$, then the space $C K_{l}$ contains no Lie subalgebra of dimension $\geq 2$.

Proof. This proposition immediately follows from Theorem 19. Indeed, in this case the intersection $C K_{l} \cap\left[C K_{l}, C K_{l}\right]$ is trivial, while $C K_{l}$ contains no two-dimensional commutative Lie subalgebra, because $S^{l}$ is a CROSS.

q.e.d.

Corollary 3. A sphere $S^{2 n-1}$ admits the space $C K_{\rho(2 n)-1}$, which is a Lie algebra if and only if $\rho(2 n)=2$ or $\rho(2 n)=4$, i.e. when $n$ is not a multiple of 4 .

Proof. If 8 divides $2 n$, then by Theorem 14, the dimension of the space $C K_{\rho(2 n)-1}$ is more than 4, and on the ground of Proposition 14, this space cannot be a Lie algebra. 
If 4 divides $2 n$, but 8 doesn't divide $2 n$, then $\rho(2 n)-1=3$ and by Proposition 13 , as the space $C K_{3}$ one can take the Lie algebra with the basis $X, Y, Z$, indicated there.

At the end, if 4 doesn't divide $2 n$, then $\rho(2 n)-1=1$, and any space $C K_{1}$ is a Lie algebra.

q.e.d.

Propositions 13, 14, and Theorem 14 immediately imply

Corollary 4. If 8 divides $2 n$, then there is a space $C K_{3}$ that is a Lie algebra (isomorphic to su(2)) which is not contained in any space $C K_{\rho(2 n)-1}$.

Propositions 13 and 14 imply

Corollary 5. 1) If 4 divides $2 n$, then there is a space $C K_{3} \subset s o(2 n)$, which is a Lie algebra, isomorphic to so(3).

2) If 8 divide $2 n$, then there exists a space $C K_{3} \subset$ so $(2 n)$, which is not a Lie algebra.

Example 4. Here we consider two examples of the spaces $C K_{3}$, where the first (respectively, the second) one is (respectively, is not) a subalgebra of $s o(2 n)$. In the Lie algebra $s o(4)$, consider the vectors

$$
\begin{gathered}
U_{1}=\left(\begin{array}{cccc}
0 & 0 & 0 & -1 \\
0 & 0 & -1 & 0 \\
0 & 1 & 0 & 0 \\
1 & 0 & 0 & 0
\end{array}\right), \quad U_{2}=\left(\begin{array}{cccc}
0 & 0 & -1 & 0 \\
0 & 0 & 0 & 1 \\
1 & 0 & 0 & 0 \\
0 & -1 & 0 & 0
\end{array}\right), \\
U_{3}=\left(\begin{array}{cccc}
0 & -1 & 0 & 0 \\
1 & 0 & 0 & 0 \\
0 & 0 & 0 & -1 \\
0 & 0 & 1 & 0
\end{array}\right) .
\end{gathered}
$$

It is easy to check that $U_{1}^{2}=U_{2}^{2}=U_{3}^{2}=-I, U_{1} U_{2}=-U_{3}, U_{2} U_{1}=U_{3}$, $U_{1} U_{3}=U_{2}, U_{3} U_{1}=-U_{2}, U_{2} U_{3}=-U_{1}, U_{3} U_{2}=U_{1}$. Therefore, the linear span of the vectors $U_{i}, 1 \leq i \leq 3$, in $s o(4)$ is a Lie subalgebra of the type $\mathrm{CK}_{3}$.

Now, consider in $s o(8)$ the vectors

$$
\widetilde{U}_{1}=\operatorname{diag}\left(U_{1},-U_{1}\right), \quad \widetilde{U}_{2}=\operatorname{diag}\left(U_{2},-U_{2}\right), \quad \widetilde{U}_{3}=\operatorname{diag}\left(U_{3},-U_{3}\right) .
$$

It is easy to check that $\widetilde{U}_{1}^{2}=\widetilde{U}_{2}^{2}=\widetilde{U}_{3}^{2}=-I$,

$$
\begin{gathered}
\widetilde{U}_{1} \widetilde{U}_{2}=\operatorname{diag}\left(-U_{3},-U_{3}\right), \widetilde{U}_{1} \widetilde{U}_{3}=\operatorname{diag}\left(U_{2}, U_{2}\right), \widetilde{U}_{2} \widetilde{U}_{3}=\operatorname{diag}\left(-U_{1},-U_{1}\right), \\
\widetilde{U}_{2} \widetilde{U}_{1}=\operatorname{diag}\left(U_{3}, U_{3}\right), \widetilde{U}_{3} \widetilde{U}_{1}=\operatorname{diag}\left(-U_{2},-U_{2}\right), \widetilde{U}_{3} \widetilde{U}_{2}=\operatorname{diag}\left(U_{1}, U_{1}\right) .
\end{gathered}
$$

Therefore, the linear span of the vectors $\widetilde{U}_{i}, 1 \leq i \leq 3$, in $s o(8)$ is a subspace of the type $C K_{3}$, but is not a Lie subalgebra. It is easy to check that the Lie algebra $C K_{3}+\left[C K_{3}, C K_{3}\right]$ is isomorphic to $s o(3) \oplus s o(3)$. 


\section{References}

[1] J.F. Adams, Vector fields on spheres, Ann. Math. 75 (1962), 603-632, MR 139178, Zbl 0112.38102.

[2] J.F. Adams, P.D. Lax \& R.S. Phillips, On matrices whose real linear combinations are non-singular, Proc. Amer. Math. Soc. 16 (1965), 318-322; Correction. Ibid. 17 (1966), 945-947 (1966), MR 179183, MR 0201460, Zbl 0168.02404.

[3] V.E. Balabaev, Construction of maximal number of linearly independent vector fields on spheres (Russian), Mat. Zametki, 67(5) (2000), 643-653, English translation in: Mat. Notes, 67(5) (2000), 547-555, MR 1822611, Zbl 0988.57016.

[4] P.E. Barbano, Automorphisms and quasi-conformal mappings of Heisenberg type groups, J. Lie Theory, 8(2) (1998), 255-277, MR 1650337, Zbl 0906.22007.

[5] V.N. Berestovskii, Homogeneous Busemann G-spaces (Russian), Sibirsk. Mat. Zh. 23(1) (1982), 3-15. English translation in: Siberian Math. J. 23(2) (1982), 141-150, Zbl 0516.53059, MR 0652219.

[6] V.N. Berestovskii \& Yu.G. Nikonorov, Killing vector fields of constant length on locally symmetric Riemannian manifolds, Transform. Groups, 13(1) (2008), 25-45, MR 2421316, Zbl pre05320883.

[7] V.N. Berestovskii \& Yu.G. Nikonorov, On $\delta$-homogeneous Riemannian manifolds, Diff. Geom. Appl., 26(5) 2008, 514-535, Zbl pre05363200.

[8] V. Berestovskii \& C. Plaut, Homogeneous spaces of curvature bounded below, J. Geom. Anal. 6(2) (1999), 203-224, MR 1759445, Zbl 1009.53038.

[9] J. Berndt, F. Tricerri \& L. Vanhecke, Generalized Heisenberg Groups and Damek-Ricci Harmonic Spaces, Lecture Notes in Mathematics 1598, Springer, Berlin, 1995, MR 1340192, Zbl 0818.53067.

[10] A.L. Besse, Manifolds all of whose Geodesics are closed, Springer-Verlag, Berlin, Heidelberg, New York, 1978, MR 0496885, Zbl 0387.53010.

[11] A.L. Besse, Einstein Manifolds, Springer-Verlag, Berlin, Heidelberg, 1987, MR 0867684, Zbl 0613.53001.

[12] É. Cartan \& J. A. Schouten, On riemannian geometries admitting an absolute parallelism, Nedrl. Akad. Wetensch. Proc. Ser. A, 29 (1926), 933-946, JFM 52.0744 .02 .

[13] G. Crandall \& J. Dódziuk, Integral structures on H-type Lie algebras, J. Lie Theory, 12(1) (2002) 69-79, MR 1885037, Zbl 1035.17018.

[14] E. Damek \& F. Ricci, A class of nonsymmetric harmonic Riemannian spaces, Bull. Amer. Math. Soc. 27 (1992), 139-142, MR 1142682, Zbl 0755.53032.

[15] J.E. D'Atri \& H.K. Nickerson, The existence of special orthonormal frames, J. Differential Geom. 2 (1968), 393-409, MR 0248682, Zbl 0179.50601.

[16] J. Dixmier, Algèbres enveloppantes (French), Gauthier-Villars Éditeur, Paris, Bruxelles, Montréal, 1974, MR 0498737, Zbl 0308.17007.

[17] P. Eberlein, Clifford algebras, Lecture notes, avialable at http://www.math.unc.edu/Faculty/pbe/publications.

[18] P. Eberlein \& J. Heber, Quarter pinched homogeneous spaces of negative curvature, Internat. J. Math. 7 (1996), 441-500, MR 1408835, Zbl 0880.53042.

[19] B. Eckmann, Gruppentheoretischer Beweis des Satzes von Hurwitz-Radon über die Komposition quadratischer Formen (German), Comment. Math. Helv. 15 (1943) 358-366, MR 0009936, Zbl 0028.10402. 
[20] H. Freudenthal, Clifford-Wolf-Isometrien symmetrischer Raume (German), Math. Ann. 150 (1963), 136-149, MR 0150701, Zbl 0189.22101.

[21] J. Gilbert \& M. Murray, Clifford algebras and Dirac operators in harmonic analysis, Cambridge Univ. Press, Cambridge, England, 1991, MR 1130821, Zbl 1143.43001.

[22] C. Gordon \& M. Kerr, New homogeneous Einstein metrics of negative Ricci curvature, Ann. Global Anal. Geom. 19(1) (2001), 75-101, MR 1824172, Zbl 0990.53054.

[23] J. Heber, On harmonic and asymptotically harmonic homogeneous spaces, Geom. Funct. Anal. 16(4) (2006), 869-890, MR 2255384, Zbl 1108.53022.

[24] S. Helgason, Totally geodesic spheres in compact symmetric spaces, Math. Ann. 165 (1966), 309-317, MR 0210043, Zbl 0142.19204.

[25] S. Helgason, Differential geometry, Lie groups and symmetric spaces, Academic Press, New York, San Francisco, London, 1978, MR 0514561, Zbl 0451.53038.

[26] A. Hurwitz, Über die Komposition der quadratischer Formen (German), Math. Ann. 88 (1922), 1-25, MR 1512117, JFM 48.1164.03.

[27] D. Husemoller, Fibre Bundles, Mc Graw-Hill Book Company, New York, 1966, MR 0229247, Zbl 0144.44804.

[28] A. Kaplan, On the geometry of groups of Heisenberg type, Bull. London Math. Soc. 15 (1983), 35-42, MR 0686346, Zbl 0521.53048.

[29] M. Kerr, A deformation of quaternionic hyperbolic space, Proc. Amer. Math. Soc. 134 (2006), 559-569, MR 2176025, Zbl 1092.53035.

[30] Sh. Kobayashi \& K. Nomizu, Foundations of differential geometry, V. 1. Interscience Publishers, New York, London, 1963, MR 0152974, Zbl 0119.37502. V. 2. Interscience Publishers, New York, London, 1969, MR 0238225, Zbl 0175.48504.

[31] B. Kostant, Holonomy and the Lie algebra of infinitesimal motions of a Riemannian manifold, Trans. Amer. Math. Soc. 80(2) (1955), 528-542, MR 0084825, Zbl 0066.16001.

[32] K. Nomizu, On local and global existence of Killing vector fields, Ann. of Math. 72(1) (1960), 105-120, MR 0119172, Zbl 0093.35103.

[33] A.L. Onishchik, Inclusion relations between transitive compact transformations groups (Russian), Proc. Moscow Math. Soc. 11 (1962), 199-242, MR 0153779, Zbl 0192.12601.

[34] V. Ozols, Clifford translations of symmetric spaces, Proc. Amer. Math. Soc. 44 (1974), 169-175, MR 0334093, Zbl 0285.53040.

[35] J. Radon, Lineare Scharen orthogonaler Matrizen (German), Abh. Math. Sem. Hamburg, 1 (1921), 1-14, JFM 48.0092.06.

[36] V.A. Toponogov, Metric structure of Riemannian spaces of nonnegative curvature containing direct lines (Russian), Sibirsk. Mat. Zh. 5(5) (1964), 1358-1369, MR 0185551, Zbl 0145.18501.

[37] J. A. Wolf, Sur la classification des variétés riemanniènnes homogènes à courbure constante, C. R. Acad. Sci. Paris, 250 (1960), pp. 3443-3445, MR 0115146, Zbl 0112.36701.

[38] J.A. Wolf, Vincent's conjecture on Clifford translations of the sphere, Comment. Math. Helv. 36 (1961), 33-41, MR 0137076, Zbl 0109.39201.

[39] J.A. Wolf, Locally symmetric homogeneous spaces, Comment. Math. Helv. 37 (1962/63), 65-101, MR 0148012, Zbl 0113.15302. 
[40] J. A. Wolf, Geodesic spheres in Grassmann manifolds, Illinois J. Math., 7 (1963), 425-446, MR 0156294, Zbl 0114.37002.

[41] J. A. Wolf, Elliptic spaces in Grassmann manifolds, Illinois J. Math., 7 (1963), 447-462, MR 0156295, Zbl 0114.37101.

[42] J. A. Wolf, On the geometry and classification of absolute parallelisms, I, J. Differential Geom. 6 (1972), 317-342, MR 0312442, Zbl 0251.53014.

[43] J. A. Wolf, On the geometry and classification of absolute parallelisms, II, J. Differential Geom. 7 (1972), 19-44, MR 0312443, Zbl 0276.53017.

[44] J.A. Wolf, Spaces of constant curvature. Third edition, Publish or Perish, Inc., Boston, Mass., 1974, MR 0343214, Zbl 0281.53034.

[45] P. Zvengrowski, Canonical vector Fields on Spheres, Comment. Math. Helv. 43 (1968), 341-347, MR 0253363, Zbl 0175.20201.

OMsk Branch of Sobolev Institute of Mathematics SD RAS

644099, Omsk, ul. Pevtsova, 13, Russia

E-mail address: berestov@ofim.oscsbras.ru

RubTsovsk Industrial Institute of Altay State Technical University

AFTER I.I. Polzunov

658207, Rubtsovsk, Traktornaya, 2/6, Russia

E-mail address: nik@inst.rubtsovsk.ru 